\begin{tabular}{|c|l|}
\hline Title & Stochastic fluctuations in the Gross Pitaevskii equation \\
\hline Author(s) & De Bouard, A nne; Fukuizumi, Reika \\
\hline Citation & Hokkaido University Preprint Series in Mathematics, 842, 1-25 \\
\hline Issue Date & 2007 \\
\hline DOI & 10.14943/83992 \\
\hline Doc URL & http://hdl.handle.net/2115/69651 \\
\hline Type & bulletin (article) \\
\hline File Information & pre842.pdf \\
\hline
\end{tabular}

Instructions for use 


\title{
STOCHASTIC FLUCTUATIONS IN THE GROSS-PITAEVSKII EQUATION
}

\author{
ANNE DE BOUARD ${ }^{1}$ AND REIKA FUKUIZUMI ${ }^{2,3}$ \\ ${ }^{1}$ CNRS, Laboratoire de Mathématiques d'Orsay, Orsay Cedex, F-91405, France; \\ Univ Paris-Sud, Orsay cedex, F-91405, France \\ Anne.debouard@math.u-psud.fr \\ 2 Department of Mathematics, Hokkaido University, Sapporo 060-0810, Japan \\ Reika.Fukuizumi@math.u-psud.fr
}

\begin{abstract}
We study from a mathematical point of view a model equation for Bose Einstein condensation, in the case where the trapping potential varies randomly in time. The model is the so called Gross-Pitaevskii equation, with a quadratic potential with white noise fluctuations in time. We prove the existence of solutions in $1 \mathrm{D}$ and $2 \mathrm{D}$ in the energy space. The blowup phenomenon is also discussed under critical and super critical nonlinear interactions in the attractive case.
\end{abstract}

\section{INTRODUCTION}

In this paper we consider the following nonlinear Schrödinger equation (named GrossPitaevskii equation in this context) perturbed by a random quadratic potential in dimension one or two :

$$
i \partial_{t} u+\left(\Delta u-V(x) u+\lambda|u|^{2 \sigma} u+i \gamma u\right)=\varepsilon K(x) u \dot{\xi}, \quad x \in \mathbb{R}^{d}, \quad d=1,2, \quad t \geq 0,
$$

where $V(x)=K(x)=|x|^{2}, \lambda= \pm 1$ and $\dot{\xi}$ is a white noise in time with correlation function $\mathbb{E}(\dot{\xi}(t) \dot{\xi}(s))=\delta_{0}(t-s)$. Here, $\delta_{0}$ denotes the Dirac measure at the origin, $\sigma>0, \gamma \geq 0$ and $\varepsilon>0$. The product arising in the right hand side is interpreted in the Stratonovich sense, since the noise here naturally arises as the limit of processes with nonzero correlation length. We moreover assume that the noise is real valued.

Equation (1.1) with $d=2, \sigma=1$ and $V(x)=K(x)=|x|^{2}$ was introduced in [1] as a model for the evolution of the wave function of a Bose-Einstein condensate trapped by a system of laser beams (see also [2]). Indeed, according to [1], fluctuations of the laser intensity should be taken into account in this case, and those fluctuations may be

1991 Mathematics Subject Classification. 35Q55, 60H15 .

Key words and phrases. Nonlinear Schrödinger equation, stochastic partial differential equations, white noise.

3 current address: Laboratoire de Mathématiques d'Orsay, Univ Paris-Sud, Orsay cedex, F-91405, France. 
regarded as fluctuations of the harmonic trap potential in the mean field approximation. Assuming that those fluctuations are delta-correlated in time, one is then lead to consider equation (1.1), in which the term $\varepsilon \dot{\xi}(t)$ represents the deviations of the laser intensity $E(t)$ around its mean value (see [1]). Also, in this model, the sign of $\lambda$ is related to the sign of the atomic scattering length, which may be positive or negative. A similar model was used in [17] in dimension three, except that the fluctuations there were not assumed to be delta-correlated. Related equations may also be found in the context of optic fibers. In [3] e.g., equation (1.1) with $V(x)=0$ and $K(x)=|x|^{2}$ is considered as a model for optical soliton propagation in fibers with random inhomogeneities.

In [1], the qualitative properties of solutions of (1.1) is studied by using the "moments method" which consists in finding (finite dimensional) evolution equations satisfied by a few integral quantities of the solutions, like e.g. energy momentum, and so on. A closed system is then found in the case where there is no damping. The solutions of this system, which is a system of stochastic differential equations, are then formally approximated in the limit where the noise tends to zero. Numerical computations are also shown in [1]. However, no really clear conclusion is made about the occurence or not of collapsing states for the condensate. Our aim in the present paper is to give a precise mathematical meaning to the solutions of (1.1) and to show the occurence of collapse in the attractive case, by the use of a virial method.

In order to state precisely the problem and our results, we will consider a probability space $(\Omega, \mathcal{F}, \mathbb{P})$ endowed with a standard filtration $\left(\mathcal{F}_{t}\right)_{t \geq 0}$ and a standard real valued Brownian motion $W(t)$ on $\mathbb{R}^{+}$associated with the filtration $\left(\mathcal{F}_{t}\right)_{t \geq 0}$. We set $\dot{\xi}=\frac{d W}{d t}$ and then consider the stochastic nonlinear Schrödinger equation:

$$
i d u+\left(\Delta u-|x|^{2} u+\lambda|u|^{2 \sigma} u+i \gamma u\right) d t=\varepsilon|x|^{2} u \circ d W,
$$

where $\circ$ stands for a Stratonovich product in the right hand side of (1.2) and $\lambda= \pm 1$. We will use the equivalent Itô equation which may be written as

$$
i d u+\left(\Delta u-|x|^{2} u+\frac{i}{2} \varepsilon^{2}|x|^{4} u+\lambda|u|^{2 \sigma} u+i \gamma u\right) d t=\varepsilon|x|^{2} u d W
$$

in order to be able to use the Itô calculus. As usual, the corrective term $\frac{i}{2} \varepsilon^{2}|x|^{4} u$ in (1.3) arises as a damping term, here with a function of $|x|^{4}$. An equation of the same kind as (1.3), but without quadratic potentials (corresponding to the case $V(x)=0$ and $K(x)=1$ ) was considered in $[11,12,13]$. The noise was there depending on both the time and space variables, with white correlations in time and coloured in space. The product in the equation was also a Stratonovich product, but the corrective term coming from the Itô correction was there considered as a perturbation of the deterministic equation, in particular when using a fixed point method in order to get the local (in time) existence of solutions. This is not possible here because the linear corrective term $|x|^{4} u$ does not live in the domain of the operator $-\underset{2}{-|x|^{2}}$. In $[11,12]$, the authors could make use 
of the Strichartz estimates established in the deterministic theory. In the present case, the linear operator, corresponding to (1.3) with $\lambda=0$ or with $\lambda=0$ and $K \equiv 0$, does not easily allow us to use a similar argument. Indeed, even if the Strichartz estimates for the deterministic Schrödinger operator with a superquadratic potential are known (see $[21,22])$, the linear operator under consideration here is not self-adjoint and in particular the $|x|^{4}$ term in the potential is a dissipative term. Also as was recalled in $[8,15]$, the evolution of the linear deterministic case with a quadratic potential is given by Mehler's formula, whose generalization to the non-deterministic case would not be so clear. For the reasons mentioned above, we consider only the one or two dimensional cases, in which the nonlinear term may be handled without making use of Strichartz estimates.

In the case where $V(x)=|x|^{2}$ and $K \equiv 0$, it is known that in the energy space (see the definition of $\Sigma$ below) equation (1.1) is locally well posed for $\lambda= \pm 1$, and globally well posed if either $\lambda=-1$ or $\lambda=1$ and $\sigma<2 / d$ (see [9, 19]). Also, blow up phonomena appear for $\lambda=1$ and $\sigma \geq 2 / d$ with a certain condition on initial data, for example, a datum with negative energy (see [7]). We here generalize these deterministic results to equation (1.3), with a condition on the initial datum, which now depends on $\varepsilon$.

We now give some notations. For $p \geq 1, L^{p}\left(\mathbb{R}^{d}\right)$ is the Lebesgue space of complex valued, $p$-th summable functions, and the inner product in the real Hilbert space $L^{2}\left(\mathbb{R}^{d}\right)$ is denoted by $\langle\cdot, \cdot\rangle$, i.e.,

$$
\langle u, v\rangle=\operatorname{Re} \int_{\mathbb{R}^{d}} u(x) \overline{v(x)} d x, \quad \text { for } \quad u, v \in L^{2}\left(\mathbb{R}^{d}\right)
$$

The norm in $L^{p}\left(\mathbb{R}^{d}\right)$ is denoted by $|\cdot|_{L^{p}}$.

We define for $s \in \mathbb{R}$ the space $H^{s}\left(\mathbb{R}^{d}\right)$ of tempered distributions $v \in \mathcal{S}^{\prime}\left(\mathbb{R}^{d}\right)$ whose Fourier transform $\hat{v}$ satisfies $\left(1+|\xi|^{2}\right)^{s / 2} \hat{v} \in L^{2}\left(\mathbb{R}^{d}\right)$. The norm in $H^{s}\left(\mathbb{R}^{d}\right)$ is denoted by $|\cdot|_{H^{s}}$. We denote the weighted space $\left\{v \in H^{1}\left(\mathbb{R}^{d}\right) ;|x| v \in L^{2}\left(\mathbb{R}^{d}\right)\right\}$ by $\Sigma$ and its norm by $|\cdot|_{\Sigma}=|\cdot|_{H^{1}}+|x \cdot|_{L^{2}}$.

If $I$ is an interval of $\mathbb{R}, E$ is a Banach space, and $1 \leq r \leq \infty$, then $L^{r}(I ; E)$ is the space of strongly Lebesgue measurable functions $v$ from $I$ into $E$ such that the function $t \rightarrow|v(t)|_{E}$ is in $L^{r}(I)$. We define similarly the spaces $C^{\alpha}(I ; E)$, or $L^{r}(\Omega ; E)$, where $\alpha$ is the Hölder exponent.

It will be convenient to approximate the stochastic nonlinear Schrödinger equation (1.2) in order to justify some formal computations. We use a cut-off function $\theta$ that is, $0 \leq \theta(\rho) \leq 1, \theta \in C_{0}^{\infty}\left(\mathbb{R}_{+}\right)$and $\theta(\rho)=0$ for $\rho \geq 2, \theta(\rho)=1$ if $0 \leq \rho \leq 1$. We define $\theta_{n}(x)=\theta\left(\frac{|x|^{2}}{n}\right)$ so that $\left|D^{\alpha} \theta_{n}\right|_{L^{\infty}}=O\left(n^{-l / 2}\right)$ for $n \in \mathbb{N},|\alpha|=l, l=1,2$. 
We define the energy

$$
H(u)=\frac{1}{2}|\nabla u|_{L^{2}}^{2}+\frac{1}{2}|x u|_{L^{2}}^{2}-\frac{\lambda}{2 \sigma+2}|u|_{L^{2 \sigma+2}}^{2 \sigma+2},
$$

which is a conserved quantity of the deterministic equation, i.e. (1.1) with $K \equiv 0$ and $V(x)=|x|^{2}$. We will look for solutions in the space $\Sigma$ since $H(u)$ is well defined in $\Sigma$, thanks to the embedding $\Sigma \subset H^{1}\left(\mathbb{R}^{d}\right) \subset L^{2 \sigma+2}\left(\mathbb{R}^{d}\right)$, if $d=1$ or 2 , and it will allow us to get an estimate for the global existence.

We will have to make use of a compactness method, and Prokhorov and Skorohod Theorems. A suitable space to get estimates allowing us to use those theorems is, in the present paper for $m \in \mathbb{N}$,

$$
\Sigma^{m}=H^{m} \cap\left\{u \in L^{2}\left(\mathbb{R}^{d}\right) ;\left(1+|x|^{2}\right)^{m / 2} u \in L^{2}\left(\mathbb{R}^{d}\right)\right\} \subset \Sigma .
$$

The dual space of $\Sigma^{m}$ in the $L^{2}$ sense, which we denote by $\Sigma^{-m}$, is

$$
\Sigma^{-m}=H^{-m}+\left\{u \in \mathcal{S}^{\prime}\left(\mathbb{R}^{d}\right) ; \frac{u}{\left(1+|x|^{2}\right)^{m / 2}} \in L^{2}\left(\mathbb{R}^{d}\right)\right\},
$$

and the norm in $\Sigma^{-m}$ is given by

$$
|u|_{\Sigma^{-m}}=\inf \left\{\left|u_{1}\right|_{H^{-m}}+\left|\frac{u_{2}}{\left(1+|x|^{2}\right)^{m / 2}}\right|_{L^{2}} ; u=u_{1}+u_{2}\right\} \quad \text { for } \quad u \in \Sigma^{-m} .
$$

The results are the following. First, we study the local well posedness of (1.3).

Theorem 1. Assume $\sigma>0, \gamma \geq 0$ and $\lambda= \pm 1$.

(i) Assume $d=1$. For any $u_{0} \in \Sigma$, there exist a stopping time $\tau^{*}\left(u_{0}, \omega\right)$ and a unique solution $u(t)$ adapted to $\left(\mathcal{F}_{t}\right)_{t>0}$ of $(1.3)$ with $u(0)=u_{0}$, which is almost surely in $C([0, \tau] ; \Sigma)$ for any $\tau<\tau^{*}\left(u_{0}\right)$. Moreover, we have almost surely,

$$
\tau^{*}\left(u_{0}, \omega\right)=+\infty \text { or } \limsup _{t / \tau^{*}\left(u_{0}, \omega\right)}|u(t)|_{\Sigma}=+\infty .
$$

(ii) Assume $d=2$ and $\sigma \leq 1$. For any $u_{0} \in \Sigma$, there exist a stopping time $\tau^{*}\left(u_{0}, \omega\right)$ and a unique solution $u(t)$ adapted to $\left(\mathcal{F}_{t}\right)_{t \geq 0}$ of $(1.3)$ with $u(0)=u_{0}$, which is almost surely continuous on $[0, \tau]$ with values in $\Sigma$ endowed with its weak topology, for any $\tau<\tau^{*}\left(u_{0}\right)$. Moreover, we have almost surely,

$$
\tau^{*}\left(u_{0}, \omega\right)=+\infty \text { or } \limsup _{t / \tau^{*}\left(u_{0}, \omega\right)}|u(t)|_{L^{2 \sigma+2}}=+\infty .
$$

As was previously mentioned, a compactness method will be used in order to prove Theorem 1. We note that the condition $u \in \Sigma$ is not sufficient to ensure that the quadratic variation of the martingale part in equation (1.3) is finite. Thus we have to use a cut-off of the diffusion coefficient. Moreover, since the equation is nonlinear, we have to use also a cut-off in the nonlinear term, thus introducing a new parameter in our sequence of approximate solutions, as is classical for stochastic partial differential equations. 
The uniqueness of the solution of (1.3) is necessary to derive the convergence in probability of the original sequence of regularized solutions. In order to obtain the convergence of the original sequence, we will make use of the following lemma, which was first used by Gyöngy and Krylov in [18].

Lemma 1.1. Let $Z_{n}$ be a sequence of random elements in a Polish space E equipped with the Borel $\sigma$ algebra. Then $Z_{n}$ converges in probability to an E-valued random element if and only if for every pair of subsequences $\left(Z_{\phi(n)}, Z_{\psi(n)}\right)$, there is a subsequence of $\left(Z_{\phi(n)}, Z_{\psi(n)}\right)$ which converges in law to a random element supported on the diagonal $\{(x, y) \in E \times E, x=y\}$.

However, some difficulty occurs in proving the uniqueness of the limit of the sequence of approximate solutions. Indeed, we do not know whether this uniqueness holds for a fixed value of the parameter corresponding to the cut-off in the nonlinear term. For that reason, we need to include the whole sequence of cut-off parameters in the approximate solution. A similar idea has been developped in [14] in which the convergence of a semi-discretized version of Equation (1.1) with $K=V=0$ is studied.

Secondly, we show the global existence of the solution of (1.3). For that purpose, we need to apply the Itô Formula to the energy $H(u)$. In this process, it is required that the reguralized solutions to (1.3) converge to a solution of (1.3) in the topology of strongly continuous functions of time with values in $\Sigma$. We note that Theorem 1 (ii), i.e. the case $d=2$, gives only the existence of a solution which is weakly continuous in time with values in $\Sigma$. To realize the strong convergence we need an estimate on the regularized solutions in a more regular space than $\Sigma$, for example $\Sigma^{2}$ in our case. We will then apply the argument of [6] and more regularity on the initial data and the nonlinearity will be required.

Theorem 2. Assume $d=2, \lambda= \pm 1, \gamma \geq 0$ and $1 / 2 \leq \sigma \leq 1$. For any $u_{0} \in \Sigma^{2}$ there is a unique solution $u(t)$ adapted to $\left(\mathcal{F}_{t}\right)_{t \geq 0}$ of $(1.3)$ with $u(0)=u_{0}$, which is almost surely in $C([0, \tau] ; \Sigma)$ for any $\tau<\tau^{*}\left(u_{0}\right)$, where $\tau^{*}$ is the same random time as in Theorem 1.

As a consequence, using an estimate for the energy, we have the following theorem concerning global existence.

Theorem 3. Assume $\sigma>0$ and $\gamma \geq 0$.

(i) Suppose that either $\lambda=-1$ or $\lambda=1$ and $\sigma<2 / d$. Assume also that $u_{0} \in \Sigma$ if $d=1$ or $u_{0} \in \Sigma^{2}$ and $\sigma \geq 1 / 2$ if $d=2$, then the solution of $(1.3)$ with $u(0)=u_{0}$ is global, i.e. $\tau^{*}\left(u_{0}\right)=+\infty$, a.s.

(ii) Suppose $\lambda=1$ and $\sigma=2 / d$. Assume that $u_{0} \in \Sigma$ if $d=1$ and $u_{0} \in \Sigma^{2}$ if $d=2$, then the solution of (1.3) with $u(0)=u_{0}$ satisfying

$$
\left|u_{0}\right|_{L^{2}}^{4 / d}<1 /\left(2 C_{d}\right)
$$


is global, i.e. $\tau^{*}\left(u_{0}\right)=+\infty$ a.s., where $C_{d}$ is the best constant in the GagliardoNirenberg Inequality

$$
\frac{1}{\frac{4}{d}+2} \int_{\mathbb{R}^{d}}|v|^{4 / d+2} d x \leq C_{d}|\nabla v|_{L^{2}}^{2}|v|_{L^{2}}^{4 / d} .
$$

Lastly, the blow up phenomenon will be discussed. In the following theorem, we only consider the case $\gamma=0$. We remark that if $d=1$ we may prove an analogous result for the case $\gamma>0$ if $\sigma>2$ (see Remark 4.2 for details).

Theorem 4. Let $\gamma=0$. Assume that $u_{0} \in \Sigma$ and $\sigma \geq 2$ if $d=1$ or $u_{0} \in \Sigma^{2}$ and $\sigma=1$ if $d=2$. Assume also that $\lambda=1$ and for some deterministic $\bar{t}>0$ with $\varepsilon^{2} \bar{t} \leq 1$,

$$
I\left(u_{0}\right)+4 G\left(u_{0}\right) \bar{t}+8 H\left(u_{0}\right) \bar{t}^{2}<0,
$$

then

$$
\mathbb{P}\left(\tau^{*}\left(u_{0}\right) \leq \bar{t}\right)>0 .
$$

For equation (1.1) with $K \equiv V \equiv 0$ and a noise which is white in time and correlated but nondegenerate in space, the authors in [13] proved that the blow-up phenomenon occurs for any nonzero initial data if $\sigma>2$. We are not able to prove such a result in the present case (see Remark 4.1), and a noise depending only on time might not have enough influence to cause the blow-up for any initial data.

The paper is organized as follows: in Section 2, we prove the local well posedness of (1.3). In Section 3, we show the global existence of the solution of (1.3), and in Section 4 we study the blow up phenomenon. To lighten notations, we denote in what follows by $C\left(u_{0}, \cdots\right)$ a constant which depends on $u_{0}$ and so on.

\section{LOCAL EXISTENCE}

This section is devoted to prove Theorems 1 and 2. We will give a detailed proof only for the case $d=2$ since the proof in the case $d=1$ is much simpler. We will just make some remarks for $d=1$ at the end of the section.

2.1. Proof of Theorem 1 (ii) $(d=2)$. First we consider an approximation of Eq.(1.3) as follows:

$$
i d u_{n}+\left(\Delta u_{n}-|x|^{2} u_{n}+\frac{i}{2} \varepsilon^{2} \theta_{n}^{2}(x)|x|^{4} u_{n}+g_{n}\left(u_{n}\right)+i \gamma u_{n}\right) d t=\varepsilon \theta_{n}(x)|x|^{2} u_{n} d W
$$

with $u_{n}(0)=u_{0}$, where

$$
g_{n}(s)=\lambda|s|^{2 \sigma} s \quad \text { if } \quad|s| \leq n, \quad g_{n}(s)=\lambda n^{2 \sigma} s \quad \text { if } \quad|s| \geq n .
$$

We remark that for all $u_{0} \in \Sigma$, there is a unique solution $u_{n} \in L^{2}(\Omega ; C([0,+\infty) ; \Sigma))$ of (2.1). This solution satisfies the following estimates which will allow us to apply a compactness method, and pass to the limit as $n$ goes to infinity. 
Lemma 2.1. Assume $\sigma>0, \lambda= \pm 1, \gamma \geq 0$ and $u_{0} \in \Sigma$. Let $u_{n}$ be the solution of (2.1). For any $T>0$ and $R>0, u_{n}$ satisfies

$$
\mathbb{E}\left(\sup _{t \in\left[0, \tau_{n}^{R} \wedge T\right]}\left|u_{n}(t)\right|_{\Sigma}^{2}\right) \leq C\left(T,\left|u_{0}\right|_{\Sigma}, R\right),
$$

where $\tau_{n}^{R}=\inf \left\{t \geq 0 ;\left|u_{n}(t)\right|_{L^{2 \sigma+2}}>R\right\}$.

Lemma 2.2. Assume $\sigma>0, \lambda= \pm 1, \gamma \geq 0$ and $u_{0} \in \Sigma$. Let $u_{n}$ be the solution of (2.1). For any $T>0$ and any real number $0 \leq \alpha<1 / 4$, there exists a constant $C$ independent of $n$ such that

$$
\mathbb{E}\left(\left|u_{n}(t)\right|_{C^{\alpha}\left(\left[0, \tau_{n}^{R} \wedge T\right] ; \Sigma^{-4}\right)}^{2}\right) \leq C\left(T,\left|u_{0}\right|_{\Sigma}, R\right)
$$

We do not prove these lemmas right here, but we will give a proof in Appendix. As already mentioned before, we will need the uniqueness of the limit sequence. We first have the following result, which is obtained exactly as in Section 3.6 of [9], by using suitable stopping times.

Lemma 2.3. Let $T>0$ be fixed. Assume $0<\sigma \leq 1, \gamma \geq 0, \lambda= \pm 1$ and $u_{0} \in \Sigma$. Let $u(t)$ and $v(t)$ be two adapted processes with paths a.s. in $L^{\infty}(0, T ; \Sigma) \cap C\left([0, T] ; L^{2}\right)$ and with $u(0)=v(0)=u_{0}$ a.s.; we assume that $u$ and $v$ are solutions of $(1.3)$ on $\left[0, \tau_{u}\right]$ and $\left[0, \tau_{v}\right]$ respectively, where $\tau_{u}$ and $\tau_{v}$ are stopping times. Then we have $u=v$ on $\left[0, \tau_{u} \wedge \tau_{v} \wedge T\right]$, a.s.

Proof. Let $w=u-v$ and $\tau=\tau_{u} \wedge \tau_{v}$. Then $w$ satisfies the following equation on $[0, \tau]$ :

$$
i d w+\left(\Delta w-|x|^{2} w\right) d t+\frac{i}{2} \varepsilon^{2}|x|^{4} w d t+i \gamma w d t+\lambda\left(|u|^{2 \sigma} u-|v|^{2 \sigma} v\right) d t=\varepsilon|x|^{2} w d W
$$

Applying the Itô formula to $|w|_{L^{2}}^{2}$ we have

$$
d|w|_{L^{2}}^{2}=2 \lambda\left\langle|u|^{2 \sigma} u-|v|^{2 \sigma} v, i w\right\rangle d t-2 \gamma|w|_{L^{2}}^{2} d t
$$

Therefore if we define the function $h \in L^{\infty}(0, T ; \Sigma)$ a.s. by

$$
h(t)=|u(t)|+|v(t)|, \quad \text { for all } t \in[0, T]
$$

then since $\sigma \leq 1$,

$$
|w(t \wedge \tau)|_{L^{2}}^{2} \leq C \int_{0}^{t \wedge \tau}\left(\int_{\mathbb{R}^{2}}\left(1+h^{2}(s)\right)|w(s)|^{2} d x\right) d s
$$

a.s. for any $t \in[0, T]$. Now we consider for $M>0$,

$$
\tilde{\tau}_{M}=\inf \left\{t, 0 \leq t \leq T,|h|_{L^{\infty}(0, t ; \Sigma)}>M, \text { or }|w|_{L^{\infty}(0, t ; \Sigma)}>M\right\}
$$

and any number $p \in(2,+\infty)$. We deduce from Hölder's and interpolation inequalities that

$$
\int_{\mathbb{R}^{2}} h^{2}|w|^{2} d x \leq\left(\int_{\mathbb{R}^{2}} h^{2 p} d x\right)^{1 / p}|w|_{L^{4}}^{4 / p}|w|_{L^{2}}^{(2 p-4) / p}
$$


a.s. for all $t \in\left[0, \tau \wedge \tilde{\tau}_{M}\right]$. Here we remark that by Trudinger inequality, there exist two positive constants $K(M)$ and $\mu(M)$ such that

$$
\int_{\mathbb{R}^{2}}\left(e^{\mu h^{2}(t)}-1\right) d x \leq K
$$

for all $t \in\left[0, \tau \wedge \tilde{\tau}_{M}\right]$. It follows from (2.3), (2.4) and the elementary inequality

$$
z^{2 p} \leq\left(\frac{p}{\mu}\right)^{p}\left(e^{\mu z^{2}}-1\right), \quad z \in \mathbb{R}
$$

that we have a.s.

$$
\int_{\mathbb{R}^{2}} h^{2}|w|^{2} d x \leq C(M) p|w|_{L^{2}}^{(2 p-4) / p}
$$

for all $t \in\left[0, \tau \wedge \tilde{\tau}_{M}\right]$. Let now $\phi(t)=|w(t)|_{L^{2}}^{2}$. Since $|\phi|_{L^{\infty}\left(0, \tau \wedge \tilde{\tau}_{M} ; \mathbb{R}\right)} \leq M^{2}$, for $p$ large enough we have $\phi(t) \leq p \phi(t)^{(p-2) / p}$ if $t \in\left[0, \tau \wedge \tilde{\tau}_{M}\right]$. We can then conclude, exactly as in $[9]$, by considering the differential inequality satisfied by

$$
\Phi_{p}(t)=\int_{0}^{t} \phi(s)^{\frac{p-2}{p}} d s
$$

on $\left[0, \tau \wedge \tilde{\tau}_{M}\right]$ that for a certain $T(M)>0$, we have

$$
\int_{0}^{T(M) \wedge \tau \wedge \tilde{\tau}_{M}} \phi(s) d s=0, \quad \text { a.s. }
$$

Thus, $w \equiv 0$ a.s. on $\left[0, T(M) \wedge \tau \wedge \tilde{\tau}_{M}\right]$. We repeat the above argument on $[T(M) \wedge \tau \wedge$ $\left.\tilde{\tau}_{M}, 2 T(M) \wedge \tau \wedge \tilde{\tau}_{M}\right]$, and so on. Finally we get $w \equiv 0$ a.s. on $\left[0, \tau \wedge \tilde{\tau}_{M}\right]$, for all $M>0$. Since $h \in L^{\infty}(0, T ; \Sigma)$ and $w \in L^{\infty}(0, T ; \Sigma)$ a.s., it follows that $\lim _{M \rightarrow+\infty} \tilde{\tau}_{M}=T$ a.s. and so, $w \equiv 0$ a.s. on $\left[0, \tau_{u} \wedge \tau_{v} \wedge T\right]$, i.e., $u=v$ a.s. on $\left[0, \tau_{u} \wedge \tau_{v} \wedge T\right]$.

We are now in position to prove Theorem 1. From now on, we always assume $\sigma>0$, $\lambda= \pm 1$ and $\gamma \geq 0$.

Let

$$
\left\{\begin{array}{clrl}
v_{n}^{R}(t) & =u_{n}(t) & & \text { for } \quad t \leq \tau_{n}^{R} \\
d v_{n}^{R}(t) & =v_{n}^{R}\left(\tau_{n}^{R}\right) d t & & \text { for } \quad t>\tau_{n}^{R},
\end{array}\right.
$$

where $u_{n}$ is a solution of (2.1) with $u_{0} \in \Sigma$, and $\tau_{n}^{R}$ is defined in Lemma 2.1.

Remark 2.1. Note that $\tau_{n}^{R}$ is equivalently defined as

$$
\tau_{n}^{R}=\inf \left\{t \geq 0 ;\left|v_{n}^{R}(t)\right|_{L^{2 \sigma+2}}>R\right\} .
$$

Indeed, for $t>\tau_{n}^{R}$,

$$
v_{n}^{R}(t)=u_{n}\left(\tau_{n}^{R}\right)+\left(t-\tau_{n}^{R}\right) u_{n}\left(\tau_{n}^{R}\right) .
$$

Thus, we have

$$
\left|v_{n}^{R}(t)\right|_{L^{2 \sigma+2}}=\left(1+t-\tau_{n}^{R}\right)\left|u_{n}\left(\tau_{n}^{R}\right)\right|_{L^{2 \sigma+2}}=\left(1+t-\tau_{n}^{R}\right) R>R .
$$


We now consider the sequence $\left\{\left(v_{n}^{R}\right)_{R \in \mathbb{N}}\right\}_{n \in \mathbb{N}}$, and set $V_{n}=\left(v_{n}^{R}\right)_{R \in \mathbb{N}}$. We then prove the following lemma concerning the tightness of the sequence of regularized solutions.

Lemma 2.4. Let $T>0$. For any pair of subsequence $(l(n), k(n))_{n \in \mathbb{N}}$, the family of laws $\left(\mathcal{L}\left(V_{l(n)}, V_{k(n)}, W\right)\right)_{n \in \mathbb{N}}$ is tight in $\left(C\left([0, T] ; L^{2 \sigma+2} \cap L^{2}\right)\right)^{\mathbb{N}} \times\left(C\left([0, T] ; L^{2 \sigma+2} \cap L^{2}\right)\right)^{\mathbb{N}} \times$ $C([0, T], \Sigma)$.

Proof of Lemma 2.4. The proof follows from Lemmas 2.1 and 2.2, standard properties of the Brownian motion $W$ whose paths are in $C^{\alpha}([0, T] ; \mathbb{R})$ with $0 \leq \alpha<1 / 2$, Markov's inequality, and Ascoli-Arzela and Tychonov Theorems.

Let us fix a pair of subsequences $(l(n), k(n))_{n \in \mathbb{N}}$. We infer from Lemma 2.4, the Prokhorov Theorem and the Skorohod Theorem, that there is a subsequence of $\left(V_{l(n)}, V_{k(n)}, W\right)$ which we still denote by the same letters, a probability space $(\tilde{\Omega}, \tilde{\mathcal{F}}, \tilde{\mathbb{P}})$ and random variables $\left(\tilde{V}_{1, n}, \tilde{V}_{2, n}, \tilde{W}_{n}\right), \quad\left(\tilde{V}_{1}, \tilde{V}_{2}, \tilde{W}\right)$ with values $C\left([0, T] ; L^{2 \sigma+2} \cap L^{2}\right)^{\mathbb{N}}$ $\times C\left([0, T] ; L^{2 \sigma+2} \cap L^{2}\right)^{\mathbb{N}} \times C([0, T] ; \mathbb{R})$, such that for any $n \in \mathbb{N}$,

$$
\mathcal{L}\left(\tilde{V}_{1, n}, \tilde{V}_{2, n}, \tilde{W}_{n}\right)=\mathcal{L}\left(V_{l(n)}, V_{k(n)}, W\right)
$$

and such that

$$
\begin{aligned}
& \tilde{V}_{j, n} \rightarrow \tilde{V}_{j} \text { as } n \rightarrow+\infty, \quad \tilde{\mathbb{P}} \text { a.s. in } C\left([0, T] ; L^{2} \cap L^{2 \sigma+2}\right)^{\mathbb{N}}, \text { for } j=1,2, \\
& \tilde{W}_{n} \rightarrow \tilde{W} \text { as } n \rightarrow+\infty, \quad \tilde{\mathbb{P}} \text { a.s. in } C([0, T] ; \mathbb{R}) .
\end{aligned}
$$

We here define

and

$$
\tilde{\mathcal{F}}_{t}=\sigma\left\{\tilde{V}_{j}(s), \tilde{W}(s), 0 \leq s \leq t, \quad j=1,2\right\}
$$

$$
\tilde{\mathcal{F}}_{t}^{n}=\sigma\left\{\tilde{V}_{j, n}(s), \tilde{W}_{n}(s), 0 \leq s \leq t, \quad j=1,2\right\} .
$$

It can be easily seen that $\tilde{W}$ and $\tilde{W}_{n}$ are standard real valued Brownian motions associated respectively with $\left(\tilde{\mathcal{F}}_{t}\right)_{t \geq 0}$ and $\left(\tilde{\mathcal{F}}_{t}^{n}\right)_{t \geq 0}$.

Setting now, for $j=1,2, \tilde{V}_{j, n}=\left(\tilde{v}_{j, n}^{R}\right)_{R \in \mathbb{N}}$, and $\tilde{V}_{j}=\left(\tilde{v}_{j}^{R}\right)_{R \in \mathbb{N}}$, we obtain from (2.5) that $\tilde{v}_{j, n}^{R}$ satisfy $(2.1)$ on $\left[0, \tilde{\tau}_{j, n}^{R}\right]$, with $W$ replaced by $\tilde{W}_{n}$ and

$$
\tilde{\tau}_{j, n}^{R}=\inf \left\{t, 0 \leq t \leq T ;\left|\tilde{v}_{j, n}^{R}(t)\right|_{L^{2 \sigma+2}}>R\right\} .
$$

Define

$$
\tilde{\tau}_{j}^{R}=\inf \left\{t, 0 \leq t \leq T ;\left|\tilde{v}_{j}^{R}(t)\right|_{L^{2 \sigma+2}}>R\right\} .
$$

We now perform the passage to the limit in (2.1).

Lemma 2.5. For any $R \in \mathbb{N}$ and $j=1,2$,

(i) $\tilde{\tau}_{j, n}^{R}$ converges to $\tilde{\tau}_{j}^{R}$ as $n \rightarrow+\infty$.

(ii) $\tilde{v}_{j}^{R}$ satisfies the equation

$$
\left\{\begin{array}{l}
i d \tilde{v}_{j}^{R}+\left(\Delta \tilde{v}_{j}^{R}-|x|^{2} \tilde{v}_{j}^{R}+\frac{i}{2} \varepsilon^{2}|x|^{4} \tilde{v}_{j}^{R}+\lambda\left|\tilde{v}_{j}^{R}\right|^{2 \sigma} \tilde{v}_{j}^{R}+i \gamma \tilde{v}_{j}^{R}\right) d t=\varepsilon|x|^{2} \tilde{v}_{j}^{R} d \tilde{W} \quad t \leq \tilde{\tau}_{j}^{R}, \\
d \tilde{v}_{j}^{R}(t)=\tilde{v}_{j}^{R}\left(\tilde{\tau}_{j}^{R}\right) d t \quad t>\tilde{\tau}_{j}^{R},
\end{array}\right.
$$


and $\tilde{v}_{j}^{R}(0)=\tilde{u_{0}}$,

(iii) $\tilde{v}_{j}^{R}(t)=\tilde{v}_{j}^{R+1}(t) \quad$ for $\quad t \leq \tilde{\tau}_{j}^{R}$.

Proof. Let $R>0$ and $j=1$ or 2 be fixed. First we begin with the proof of (i). Since $\tilde{\tau}_{j, n}^{R} \in[0, T]$ for any $n \in \mathbb{N}$, there exists a subsequence $\left\{\tilde{\tau}_{j, n_{k}}^{R}\right\}$ such that $\tilde{\tau}_{j, n_{k}}^{R} \rightarrow \tau_{R}$ as $k \rightarrow \infty$. If $t<\tau_{R}$, we have $\left|\tilde{v}_{j, n_{k}}^{R}(t)\right|_{L^{2 \sigma+2}} \leq R$ for sufficiently large $k$; thus, since $\tilde{v}_{j, n_{k}}^{R} \rightarrow \tilde{v}_{j}^{R}$ in $C\left([0, T] ; L^{2 \sigma+2}\right)$, we conclude $\left|\tilde{v}_{j}^{R}(t)\right|_{L^{2 \sigma+2}} \leq R$ which implies $\tilde{\tau}_{j}^{R} \geq \tau_{R}$. On the other hand, if $t>\tau_{R}$, we can say that for sufficiently large $k, t \geq \tilde{\tau}_{j, n_{k}}^{R}+\varepsilon$, where $\varepsilon=\left(t-\tau_{R}\right) / 2>0$. Therefore,

$$
\left|\tilde{v}_{j, n_{k}}^{R}(t)\right|_{L^{2 \sigma+2}}=\left(1+t-\tilde{\tau}_{j, n_{k}}^{R}\right)\left|\tilde{v}_{j, n_{k}}^{R}\left(\tilde{\tau}_{j, n_{k}}^{R}\right)\right|_{L^{2 \sigma+2}} \geq(1+\varepsilon) R>R
$$

Then, letting $k$ to $+\infty$, we have

$$
\left|\tilde{v}_{j}^{R}(t)\right|_{L^{2 \sigma+2}} \geq(1+\varepsilon) R>R,
$$

and so $t \geq \tilde{\tau}_{j}^{R}$. Accordingly, $\tau_{R}=\tilde{\tau}_{j}^{R}$ and in the same way all converging subsequences converge to $\tilde{\tau}_{j}^{R}$.

Secondly, since $\tilde{v}_{j, n}^{R}$ converges to $\tilde{v}_{j}^{R}$ in $C\left([0, T] ; L^{2} \cap L^{2 \sigma+2}\right)$ a.s. as $n \rightarrow+\infty$ and $\tilde{v}_{j, n}^{R}$ satisfies (2.2), we can prove (ii), letting $n \rightarrow+\infty$ in (2.1) and using Remark 2.1. The fact (iii) follows in a similar way to Lemma 4.1 in [11].

Adding a point $\Delta$ at infinity in $L^{2 \sigma+2}\left(\mathbb{R}^{d}\right)$ with the topology such that neighborhoods of $\{\Delta\}$ in $L^{2 \sigma+2}\left(\mathbb{R}^{d}\right) \cup\{\Delta\}$ are defined as complements of closed balls of $L^{2 \sigma+2}\left(\mathbb{R}^{d}\right)$, we set

$$
\tilde{v}_{j}(t)=\left\{\begin{aligned}
\tilde{v}_{j}^{R}(t) & \text { if there exists } R \text { such that } t<\tilde{\tau}_{j}^{R} \\
\Delta & \text { if } t \geq \lim \sup _{R \rightarrow \infty} \tilde{\tau}_{j}^{R}=\tilde{\tau}_{j}^{*} .
\end{aligned}\right.
$$

Similarly, we put

$$
v_{n}(t)=v_{n}^{R}(t), \quad \text { if } \quad t<\tau_{n}^{R} .
$$

Note that in the above definition, such a $R$ always exists since $v_{n} \in L^{\infty}(0, T ; \Sigma)$. We define in the same way

$$
\tilde{v}_{j, n}(t)=\tilde{v}_{j, n}^{R}(t), \quad \text { if } \quad t<\tilde{\tau}_{j, n}^{R} .
$$

Also, for any $R>0$ and any $j=1,2$, and for a.e. $\omega \in \Omega, \tilde{v}_{j, n}^{R} \in C([0, T] ; \Sigma)$ and $\left|\tilde{v}_{j, n}^{R}\right|_{L^{\infty}(0, T ; \Sigma)} \leq C(\omega)$; hence $\tilde{v}_{j, n}^{R}$ converges in $L^{\infty}(0, T ; \Sigma)$ weak star to $\tilde{v}_{j}^{R} \in L^{\infty}(0, T ; \Sigma)$ a.s. It follows from Lemma 2.3 (note that here we need $\sigma \leq 1$ ) and Lemma 2.5 that $\tilde{v}_{1}^{R}=\tilde{v}_{2}^{R}$ on $\left[0, \tilde{\tau}_{1}^{R} \wedge \tilde{\tau}_{2}^{R}\right]$ for each $R>0$, hence $\tilde{\tau}_{1}^{*}=\tilde{\tau}_{2}^{*}$ and $\tilde{v}_{1}(t)=\tilde{v}_{2}(t)$ on $[0, T]$, a.s.

In order to apply Lemma 1.1 to the sequence $v_{n}$, we shall prove the following lemma.

Lemma 2.6. Let $T>0$ be fixed. Then $\tilde{v}_{j, n}$ converges to $\tilde{v}_{j}$ in probability in $C\left([0, T] ; L^{2 \sigma+2} \cup\{\Delta\}\right)$.

Remark 2.2. The space $L^{2 \sigma+2}\left(\mathbb{R}^{d}\right) \cup\{\Delta\}$ with the topology defined above is actually a metric space, since we may endow it with the distance induced by the "stereographic 
projection". Indeed, let $\phi$ be the mapping from $L^{2 \sigma+2}\left(\mathbb{R}^{d}\right) \cup\{\Delta\}$ into $L^{2 \sigma+2}\left(\mathbb{R}^{d}\right) \times \mathbb{R}$ defined by

$$
\phi(v)=\left(\frac{2 v}{1+|v|_{L^{2 \sigma+2}}^{2}}, \frac{|v|_{L^{2 \sigma+2}}^{2}-1}{1+|v|_{L^{2 \sigma+2}}^{2}}\right)
$$

if $v \in L^{2 \sigma+2}\left(\mathbb{R}^{d}\right)$, and $\phi(\Delta)=(0,1)$. Then it is not difficult to check that $\phi$ is bijective and bicontinuous from $L^{2 \sigma+2}\left(\mathbb{R}^{d}\right) \cup\{\Delta\}$ into $\left\{(v, \lambda) \in L^{2 \sigma+2}\left(\mathbb{R}^{d}\right) \times \mathbb{R},|v|_{L^{2 \sigma+2}}^{2}+\lambda^{2}=1\right\}$. It suffices then to define the distance $d(\bar{u}, \bar{v})=\|\phi(\bar{u})-\phi(\bar{v})\|_{L^{2 \sigma+2} \times \mathbb{R}}$, for $\bar{u}$ and $\bar{v}$ in $L^{2 \sigma+2}\left(\mathbb{R}^{d}\right) \cup\{\Delta\}$.

Proof of the Lemma. For fixed $\varepsilon \in(0,1]$, we show that

$$
\mathbb{P}\left(\sup _{t \in[0, T]} d\left(\tilde{v}_{j, n}(t), \tilde{v}_{j}(t)\right)>\varepsilon\right) \rightarrow 0, \quad \text { as } n \rightarrow+\infty,
$$

where $d$ is the distance defined in Remark 2.2. We set $\tau^{*}=\tilde{\tau}_{1}^{*}=\tilde{\tau}_{2}^{*}$. Then, we have,

$$
\begin{aligned}
& \mathbb{P}\left(\sup _{t \in[0, T]} d\left(\tilde{v}_{j, n}(t), \tilde{v}_{j}(t)\right)>\varepsilon\right) \\
& \leq \mathbb{P}\left(\sup _{t<\tau^{*}} d\left(\tilde{v}_{j, n}(t), \tilde{v}_{j}(t)\right)>\varepsilon\right)+\mathbb{P}\left(\sup _{t \geq \tau^{*}} d\left(\tilde{v}_{j, n}(t), \Delta\right)>\varepsilon\right) \\
& =\mathrm{I}+\mathrm{II} .
\end{aligned}
$$

We fix $R$ large enough so that

$$
\left\{v \in L^{2 \sigma+2}\left(\mathbb{R}^{d}\right),|v|_{L^{2 \sigma+2}}>R\right\} \subset\left\{v \in L^{2 \sigma+2}\left(\mathbb{R}^{d}\right), d(v, \Delta)<\varepsilon / 2\right\} .
$$

Then we may estimate I as follows.

$$
\begin{aligned}
\mathrm{I} & =\mathbb{P}\left(\sup _{t<\tau^{*}} d\left(\tilde{v}_{j, n}(t), \tilde{v}_{j}(t)\right)>\varepsilon\right) \\
& \leq \mathbb{P}\left(\sup _{t<\tilde{\tau}_{j}^{2 R}} d\left(\tilde{v}_{j, n}(t), \tilde{v}_{j}(t)\right)>\varepsilon\right)+\mathbb{P}\left(\sup _{\tilde{\tau}_{j}^{2 R} \leq t \leq \tau^{*}} d\left(\tilde{v}_{j, n}(t), \Delta\right)>\frac{\varepsilon}{2}\right) \\
& =(\mathrm{I} .1)+(\mathrm{I} .2) .
\end{aligned}
$$

We first consider the term (I.1). Since $\tilde{v}_{j}(t)=\tilde{v}_{j}^{2 R}(t)$ for $t \leq \tilde{\tau}_{j}^{2 R}$,

$$
\begin{aligned}
(\mathrm{I} .1) & =\mathbb{P}\left(\sup _{t \leq \tilde{\tau}_{j}^{2 R}} d\left(\tilde{v}_{j, n}(t), \tilde{v}_{j}^{2 R}(t)\right)>\varepsilon\right) \\
& \leq \mathbb{P}\left(\sup _{t \leq \tilde{\tau}_{j}^{2 R}}\left|\tilde{v}_{j, n}(t)-\tilde{v}_{j}^{2 R}(t)\right|_{L^{2 \sigma+2}}>\delta(\varepsilon)\right),
\end{aligned}
$$

for some $\delta(\varepsilon)$ with $0<\delta(\varepsilon) \leq 1$, since none of the terms $\tilde{v}_{j, n}$ and $\tilde{v}_{j}^{2 R}$ attains the value $\Delta$.

Let us now set

$$
\hat{\tau}_{j, n}^{R}=\inf \left\{t \geq 0 ;\left|\tilde{v}_{j, n}(t)-\tilde{v}_{j}^{2 R}(t)\right|_{L^{2 \sigma+2}}>\delta(\varepsilon)\right\}
$$


Then, we obtain

$$
(\mathrm{I} .1) \leq \mathbb{P}\left(\sup _{t \leq \tilde{\tau}_{j}^{2 R} \wedge \hat{\tau}_{j, n}^{R}}\left|\tilde{v}_{j, n}(t)-\tilde{v}_{j}^{2 R}(t)\right|_{L^{2 \sigma+2}} \geq \delta(\varepsilon)\right) .
$$

Furthermore, we remark that $\tilde{v}_{j, n}(t)=\tilde{v}_{j, n}^{2 R+1}(t)$ on $\left[0, \tilde{\tau}_{j}^{2 R} \wedge \hat{\tau}_{j, n}^{R}\right]$, since

$$
\left|\tilde{v}_{j, n}(t)-\tilde{v}_{j}^{2 R}(t)\right|_{L^{2 \sigma+2}} \leq \delta(\varepsilon) \leq 1 .
$$

Therefore, finally,

$$
(\text { I.1 }) \leq \mathbb{P}\left(\sup _{t \leq \tilde{\tau}_{j}^{2 R} \wedge \hat{\tau}_{j, n}^{R}}\left|\tilde{v}_{j, n}^{2 R+1}(t)-\tilde{v}_{j}^{2 R}(t)\right|_{L^{2 \sigma+2}} \geq \delta(\varepsilon)\right),
$$

and this quantity goes to 0 as $n$ goes to infinity, due to Lemma 2.5 (iii) and the fact that $\tilde{v}_{j, n}^{2 R+1}$ converges to $\tilde{v}_{j}^{2 R+1}$ a.s. in $C\left([0, T] ; L^{2 \sigma+2}\right)$.

On the other hand, by the choice of $R$,

$$
(\mathrm{I} .2) \leq \mathbb{P}\left(\sup _{\tilde{\tau}_{j}^{2 R} \leq t \leq \tau^{*}}\left|\tilde{v}_{j, n}(t)\right|_{L^{2 \sigma+2}} \leq R\right) \leq \mathbb{P}\left(\tilde{\tau}_{j}^{2 R} \leq \tilde{\tau}_{j, n}^{R}\right) .
$$

But the right hand side tends to 0 as $n$ goes to infinity, since $\lim _{n \rightarrow \infty} \tilde{\tau}_{j, n}^{R}=\tilde{\tau}_{j}^{R}<\tilde{\tau}_{j}^{2 R}$ a.s.

We have shown that $\tilde{v}_{j, n}$ converges to $\tilde{v}_{j}$ in probability in $C\left([0, T] ; L^{2 \sigma+2} \cup\{\Delta\}\right)$. Thus, $\left(\tilde{v}_{1, n}, \tilde{v}_{2, n}\right)$ converges in probability to $\left(\tilde{v}_{1}, \tilde{v}_{2}\right)$ which is supported on the diagonal, since $\tilde{v}_{1}=\tilde{v}_{2}$. By Lemma 1.1, $v_{n}$ converges to $v$ in probability. It is easy to check that $v$ is a solution of $(1.3)$ with $\tilde{W}$ replaced by $W$ on $\left[0, \tau^{*}\right)$, where $\tau^{*}=\lim \sup _{R \rightarrow+\infty} \inf \{t \geq$ $0 ;|v(t)|_{L^{2 \sigma+2}}>R$ \}. Moreover, for any $\tau<\tau^{*}$, a.s., it is easily seen by the same arguments as above that $v$ has paths in $L^{\infty}(0, \tau ; \Sigma) \cap C\left([0, \tau] ; L^{2}\left(\mathbb{R}^{d}\right)\right)$ a.s., hence its paths are a.s. continuous in time with values in $\Sigma$ endowed with its weak topology.

2.2. Proof of Theorem 2. Since we may prove Theorem 2 in a similar way to the previous subsection, we only mention some key points. The problem in the previous subsection was that we could not have the strong convergence of the regularized solution $\tilde{v}_{j, n}^{R}$ in $C([0, T] ; \Sigma)$, since it is not easy to have an estimate for $u_{n}$ in $L^{2}\left(\Omega ; C\left(\left[0, \tau_{n}^{R} \wedge T\right] ; \Sigma^{2}\right)\right)$ due to the nonlinearity. However, if we assume that $u_{0} \in \Sigma^{2}$ and $1 / 2 \leq \sigma \leq 1$, we can obtain the following estimates, applying the Itô formula to $1+\log \left(1+\left|u_{n}\right|_{\Sigma^{2}}^{2}\right)$ and using the Brezis-Gallouet inequality (see [6]). The next Lemma is proved in the Appendix.

Lemma 2.7. Assume $1 / 2 \leq \sigma \leq 1, \lambda= \pm 1, \gamma \geq 0$ and $u_{0} \in \Sigma^{2}$. Let $u_{n}$ be the solution of (2.1). For any $T>0$ and $N>0, u_{n}$ satisfies

$$
\mathbb{E}\left(\sup _{t \in\left[0, \hat{\tau}_{n}^{N} \wedge T\right]}\left(1+\log \left(1+\left|u_{n}(t)\right|_{\Sigma^{2}}^{2}\right)\right)\right) \leq C\left(T,\left|u_{0}\right|_{\Sigma^{2}}, N\right),
$$


where

$$
\hat{\tau}_{n}^{N}=\inf \left\{t \geq 0 ;\left|u_{n}(t)\right|_{\Sigma}>N\right\} .
$$

Note that it can also easily be proved as in Lemma 2.2 that

$$
\mathbb{E}\left(\left|u_{n}(t)\right|_{C^{\alpha}\left(\left[0, \hat{r}_{n}^{N} \wedge T\right] ; \Sigma^{-4}\right)}^{2}\right) \leq C\left(T,\left|u_{0}\right|_{L^{2}}, N\right)
$$

where $0<\alpha<1 / 4$.

As previously, we apply the compactness methods to the sequence $\left\{\left(v_{n}^{N}\right)_{N \in \mathbb{N}}\right\}_{n \in \mathbb{N}}$, which is defined by

$$
\left\{\begin{array}{cl}
v_{n}^{N}(t)=u_{n}(t) & \text { for } \quad t \leq \hat{\tau}_{n}^{N} \\
d v_{n}^{N}(t)=v_{n}^{N}\left(\hat{\tau}_{n}^{N}\right) d t & \text { for } \quad t>\hat{\tau}_{n}^{N} .
\end{array}\right.
$$

In fact, the estimate (2.10) allows us to have the tightness of the law of $\left\{\left(v_{n}^{N}\right)_{N \in \mathbb{N}}\right\}_{n \in \mathbb{N}}$ since, for any $N>0$ and $r>0$, we have

$$
\mathbb{P}\left(\sup _{t \in\left[0, \hat{t}_{n}^{N} \wedge T\right]}\left|v_{n}^{N}(t)\right|_{\Sigma^{2}}>r\right) \leq \frac{\mathbb{E}\left(\sup _{t \in\left[0, \hat{r}_{n}^{N} \wedge T\right]}\left(1+\log \left(1+\left|v_{n}^{N}\right|_{\Sigma^{2}}^{2}\right)\right)\right)}{1+\log \left(1+r^{2}\right)} \leq \frac{C\left(T,\left|u_{0}\right|_{\Sigma^{2}}, N\right)}{1+\log \left(1+r^{2}\right)} .
$$

Thus, for any $\varepsilon>0$ and $N>0$, taking $r(N)$ sufficiently large, the set

$$
K^{N}=\left\{v \in C\left([0, T] ; \Sigma^{2}\right) ;|v|_{L^{\infty}\left(0, T ; \Sigma^{2}\right)} \leq r(N),|v|_{C^{\alpha}\left([0, T] ; \Sigma^{-4}\right)} \leq r(N)\right\}
$$

is compact in $C([0, T] ; \Sigma)$ and $\mathbb{P}\left(v_{n}^{N} \notin K^{N}\right) \leq \varepsilon$, the result follows from Tychonov's Theorem.

Then, there exist $\tilde{v}_{j, n}^{N}$ and $\tilde{v}_{j}^{N}$ such that $\tilde{v}_{j, n}^{N}$ converges to $\tilde{v}_{j}^{N}$ a.s. in $C([0, T] ; \Sigma)$ for $j=1,2$ and each $N \in \mathbb{N}$, and $\tilde{v}_{j}^{N}$ satisfies equation (1.3) for $0 \leq t \leq \tilde{\hat{\tau}}_{j}^{N}=\lim _{n \rightarrow \infty} \tilde{\hat{\tau}}_{j, n}^{N}$. These stopping times correspond to $\tilde{v}_{j}^{N}$ and $\tilde{v}_{j, n}^{N}$ respectively, as in (2.6) and (2.7).

For all $N>0$, we define

$$
\tilde{v}_{j}(t)= \begin{cases}\tilde{v}_{j}^{N}(t) & \text { if there exists } N \text { such that } t<\tilde{\hat{\tau}}_{j}^{N}, \\ \Delta & \text { if } t \geq \lim \sup _{N \rightarrow \infty} \tilde{\hat{\tau}}_{j}^{N} .\end{cases}
$$

We see that $\tilde{v}_{j, n}$, which is defined by $\tilde{v}_{j, n}(t)=\tilde{v}_{j, n}^{N}(t)$ for $t \leq \tilde{\tau}_{j, n}^{N}$, converges to $\tilde{v}_{j}$ in probability in $C([0, T] ; \Sigma \cup\{\Delta\})$.

Then by Lemma 1.1, we get as in Section 2.1 a unique solution $v$ of (1.3) with paths a.s. in $C([0, \tau] ; \Sigma)$ for any $\tau<\tau^{* *}$, where $\tau^{* *}=\lim \sup _{N \rightarrow+\infty} \hat{\tau}^{N}$ and

$$
\hat{\tau}^{N}=\inf \left\{t \geq 0 ;|v(t)|_{\Sigma}>N\right\},
$$

Lastly we remark that $\tau^{* *}=\tau^{*}$ a.s. The fact that $\tau^{* *} \leq \tau^{*}$ simply follows from the embedding $\Sigma \subset L^{2 \sigma+2}\left(\mathbb{R}^{d}\right)$; on the other hand, we can show

$$
\mathbb{E}\left(\sup _{t \in\left[0, \tau^{R} \wedge T\right]}|v(t)|_{\Sigma}^{2}\right) \leq C\left(\left|u_{0}\right|_{\Sigma}, R, T\right),
$$


(see the proof of Lemma 2.1.) This proves, using Markov's inequality that

$$
\begin{aligned}
\mathbb{P}\left(\tau^{R}>\hat{\tau}^{N}\right) & \leq \mathbb{P}\left(\sup _{t \in\left[0, \tau^{R}\right]}|v(t)|_{\Sigma}>N\right) \\
& \leq \frac{1}{N^{2}} C\left(\left|u_{0}\right|_{\Sigma}, R, T\right) .
\end{aligned}
$$

Hence letting $N$ go to infinity, we get $\mathbb{P}\left(\tau^{R}>\tau^{* *}\right)=0$ for any $R$, thus $\tau^{*}=\tau^{* *}$ a.s.

2.3. Remarks for the case $d=1$. The verification for the case $d=1$ being much simpler, we briefly note how we may establish Theorem 1 (i).

We first solve the stochastic equation with linear drift:

$$
i d u-i A u d t=x^{2} u d W
$$

where

$$
A=i\left(\partial_{x}^{2}-x^{2}\right)-\frac{1}{2} x^{4}-\gamma,
$$

using a compactness method; for example, we employ a cut-off in the quadratic potential. As a priori estimate, using Martingale inequalities for the stochastic integrals, we can show that for an approximate solution $u_{n}(t)$ with $u_{0} \in D(A)$ and for any $T>0$, there exists a constant $C(T)$ independent of $n$ such that

$$
\mathbb{E}\left(\sup _{t \in[0, T]}\left|u_{n}(t)\right|_{\Sigma^{2}}^{2}\right) \leq C(T)\left|u_{0}\right|_{\Sigma^{2}}^{2} .
$$

It follows that the sequence of the laws of $u_{n}$ is tight in $C([0, T] ; \Sigma)$. Then, Prokhorov and Skorohod Theorems, together with the uniqueness of solutions of (2.12), lead to the existence of a strong solution of (2.12) in the probabilistic sense. Note that here, the uniqueness of solutions is easily verified for any $\sigma>0$ since $\Sigma \subset L^{\infty}(\mathbb{R})$.

Moreover, it is not difficult, by approximating the initial data, to extend this existence result to any $u_{0} \in \Sigma$, and more generally to any initial state of the form $u(s)=u_{s}$, with $u_{s}$ measurable with respect to $\mathcal{F}_{s}$ with values in $\Sigma$, a.s. In this way, we define a random propagator $U(t, s, \omega)$ by assigning to $U(t, s,.) u_{s}$ the value at time $t$ of the unique solution of (2.12) with $u(s)=u_{s}$.

Next we solve the following equation, using a fixed point argument in $L^{2}(\Omega ; C([0, T] ; \Sigma))$ and a cut-off for the nonlinear term :

$$
u(t)=U(t, 0) u_{0}+i \int_{0}^{t} U(t, s)\left[\theta\left(\frac{|u(s)|_{\Sigma}}{R}\right)|u(s)|^{2 \sigma} u(s)\right] d s,
$$

and we obtain a solution as stated in Theorem 1 (i). 


\section{Global existence}

In this section we study the global existence. Let $T>0$ be arbitrary and $u(t)$ be a solution obtained by Theorem 1 (i) if $d=1$, and Theorem 2 if $d=2$. In order to prove Theorem 3, we verify that $\tau^{*}=T$ almost surely. It suffices to show that there exists a constant $C=C\left(T, u_{0}\right)>0$, independent of $R$, such that

$$
\mathbb{E}\left(\sup _{t \in\left[0, \tau^{R} \wedge T\right]}|u(t)|_{\Sigma}\right) \leq C,
$$

where here

$$
\tau^{R}=\inf \left\{t \in\left[0, \tau^{*} \wedge T\right) ;|u(t)|_{\Sigma}>R\right\}
$$

it will then follow from Markov's inequality that $\lim _{R \rightarrow+\infty} \mathbb{P}\left(\tau^{R}<T\right)=0$, so $\tau^{*}=T$ almost surely. We first give a proof of Theorem 3 (i).

Proof of Theorem 3 (i). The two following lemmas will be useful to obtain the estimate (3.1).

Lemma 3.1. Let $\sigma, \gamma, \lambda$ and $u_{0}$ be as in Theorem $1(i)$ and $(i i)$. For any $t<\tau^{*}\left(u_{0}\right)$, we have,

$$
|u(t)|_{L^{2}} \leq\left|u_{0}\right|_{L^{2}}, \quad \text { a.s. },
$$

where $u(t)$ is the solution given by Theorem $1(i)$ and $(i i)$ with $u(0)=u_{0}$.

Lemma 3.1 is a consequence of the Itô formula applied to (1.3). The computation may be justified by an idea similar to that of [12], using a cut-off and regularization argument.

Lemma 3.2. Let $u \in \Sigma$. We assume $\sigma<2 / d$ if $\lambda=1$. Then there exists a constant $C>0$ such that

$$
|u|_{\Sigma}^{2} \leq 2 H(u)+C \beta(\lambda)|u|_{L^{2}}^{\tilde{\sigma}} .
$$

Here $\tilde{\sigma}=2\{(2-d) \sigma+2\} /(2-\sigma d)>2$ and

$$
\beta(\lambda)= \begin{cases}1, & \text { if } \lambda=1 \\ 0, & \text { if } \lambda=-1\end{cases}
$$

Proof. This is a standard consequence of the Gagliardo-Nirenberg inequality.

We now get an estimate on the energy evolution. If $\tau$ is a stopping time with $\tau \leq \tau^{R}$ almost surely, we have by a formal application of the Itô formula,

$$
\begin{aligned}
H(u(\tau))= & H\left(u_{0}\right)-2 \varepsilon \operatorname{Im} \int_{0}^{\tau} \int_{\mathbb{R}^{d}} \nabla u \cdot x \bar{u} d x d W(s)+2 \varepsilon^{2} \int_{0}^{\tau}|x u|_{L^{2}}^{2} d s \\
& -\gamma \int_{0}^{\tau}|u|_{\Sigma}^{2} d s+\lambda \gamma \int_{0}^{\tau}|u|_{L^{2 \sigma+2}}^{2 \sigma+2} d s .
\end{aligned}
$$

This formal computation may be justified by using a cut-off and regularizing procedure as in [12]. However, in order to obtain the equality in (3.3), we need the strong convergence in $C([0, T] ; \Sigma)$ of the sequence of regularized solutions, which is the case if $u_{0} \in \Sigma^{2}$ and 
$1 / 2 \leq \sigma \leq 1$ for $d=2$, as shows the proof of Theorem 2. We note that the stochastic integral in the right hand side of (3.3) is a square integrable real valued martingale since

$$
\int_{0}^{\tau^{R} \wedge T}\left|\operatorname{Im} \int_{\mathbb{R}^{d}} \nabla u \cdot x \bar{u} d x\right|^{2} d s \leq C T \sup _{0 \leq t \leq \tau^{R} \wedge T}\left(|\nabla u|_{L^{2}}^{4}+|x u|_{L^{2}}^{4}\right) \leq C T R, \quad \text { a.s. }
$$

We first estimate the expectation of the energy. For a stopping time $\tau<\tau^{R}$, we have, using (3.3) and Lemma 3.2,

$$
\begin{aligned}
\mathbb{E}(H(u(\tau)))= & H\left(u_{0}\right)+2 \varepsilon^{2} \mathbb{E}\left(\int_{0}^{\tau}|x u|_{L^{2}}^{2} d s\right)-\gamma \mathbb{E}\left(\int_{0}^{\tau}|u|_{\Sigma}^{2} d s\right)+\lambda \gamma \mathbb{E}\left(\int_{0}^{\tau}|u|_{L^{2 \sigma+2}}^{2 \sigma+2} d s\right) \\
\leq & H\left(u_{0}\right)+2 \varepsilon^{2} \int_{0}^{T} \mathbb{E}\left(\mathbb{1}_{[0, \tau]}|u|_{\Sigma}^{2}\right) d s+C \gamma \beta(\lambda) \mathbb{E}\left(\int_{0}^{\tau}\left(|\nabla u|_{L^{2}}^{2}+|u|_{L^{2}}^{\tilde{\sigma}}\right) d s\right) \\
\leq & H\left(u_{0}\right)+C \varepsilon^{2} \int_{0}^{T} \mathbb{E}\left(H\left(\mathbb{1}_{[0, \tau]} u(s)\right)+\beta(\lambda) \mathbb{1}_{[0, \tau]}|u|_{L^{2}}^{\tilde{\sigma}}\right) d s \\
& \quad+C \gamma \beta(\lambda) \mathbb{E}\left(\int_{0}^{T}\left(\mathbb{1}_{[0, \tau]}|\nabla u|_{L^{2}}^{2}+\mathbb{1}_{[0, \tau]}|u|_{L^{2}}^{\tilde{\sigma}}\right) d s\right) \\
\leq & H\left(u_{0}\right)+\beta(\lambda) T C(\varepsilon, \gamma)\left|u_{0}\right|_{L^{2}}^{\tilde{\sigma}}+C(\varepsilon, \beta(\lambda), \gamma) \int_{0}^{T} \mathbb{E}\left(H\left(\mathbb{1}_{[0, \tau]} u(s)\right)\right) d s,
\end{aligned}
$$

where we have used Lemma 3.1 in the last inequality. Thus we have, by the Gronwall Lemma,

$$
\begin{aligned}
\mathbb{E}\left(H\left(\mathbb{1}_{[0, \tau]} u(t)\right)\right) & \leq\left(C+H\left(u_{0}\right)+\beta(\lambda) T C(\varepsilon, \gamma)\left|u_{0}\right|_{L^{2}}^{\tilde{\sigma}}\right) e^{C T} \\
& =C\left(\varepsilon, \gamma, T, H\left(u_{0}\right),\left.\beta(\lambda)\left|u_{0}\right|\right|_{L^{2}} ^{\tilde{\sigma}}\right) .
\end{aligned}
$$

Next, we have from (3.3)

$$
\begin{aligned}
\mathbb{E}\left(\sup _{t \in\left[0, \tau^{R} \wedge T\right]} H(u(t))\right) \leq & H\left(u_{0}\right)+2 \varepsilon \mathbb{E}\left(\sup _{t \in\left[0, \tau^{R} \wedge T\right]}\left|\int_{0}^{t} \int_{\mathbb{R}^{d}} \nabla u \cdot x \bar{u} d x d W(s)\right|\right) \\
& +2 \varepsilon^{2} \mathbb{E}\left(\int_{0}^{\tau^{R} \wedge T}|x u|_{L^{2}}^{2} d s\right)+\beta(\lambda) \gamma \mathbb{E}\left(\int_{0}^{\tau^{R} \wedge T}|u|_{L^{2 \sigma+2}}^{2 \sigma+2} d s\right) .
\end{aligned}
$$

It follows from a standard martingale inequality that

$$
\mathbb{E}\left(\sup _{t \in\left[0, \tau^{R} \wedge T\right]}\left|\int_{0}^{t}\left(\int_{\mathbb{R}^{d}} \nabla u \cdot x \bar{u} d x\right) d W(s)\right|^{2}\right) \leq 4 \sup _{t \in[0, T]} \mathbb{E}\left(\left|\int_{0}^{t \wedge \tau^{R}}\left(\int_{\mathbb{R}^{d}} \nabla u \cdot x \bar{u} d x\right) d W(s)\right|^{2}\right) .
$$


Using (3.6) and the Cauchy-Schwarz inequality,

$$
\begin{aligned}
& \mathbb{E}\left(\sup _{t \in\left[0, \tau^{R} \wedge T\right]} H(u(t))\right) \leq H\left(u_{0}\right)+C \varepsilon\left(\mathbb{E}\left(\int_{0}^{T \wedge \tau^{R}}\left(|\nabla u|_{L^{2}}^{4}+|x u|_{L^{2}}^{4}\right) d s\right)\right)^{1 / 2} \\
& \quad+2 \varepsilon^{2} \mathbb{E}\left(\int_{0}^{\tau^{R} \wedge T}|x u|_{L^{2}}^{2} d s\right)+\beta(\lambda) \gamma \mathbb{E}\left(\int_{0}^{\tau^{R} \wedge T}|u|_{L^{2 \sigma+2}}^{2 \sigma+2} d s\right) \\
& \left.\leq H\left(u_{0}\right)\right)+C \varepsilon\left(\int_{0}^{T} \mathbb{E}\left(\mathbb{1}_{\left[0, \tau^{R} \wedge T\right]}|u(s)|_{\Sigma}^{4}\right) d s\right)^{1 / 2} \\
& +2 \varepsilon^{2} \mathbb{E}\left(\int_{0}^{\tau^{R} \wedge T}|x u|_{L^{2}}^{2} d s\right)+C \beta(\lambda) \gamma \mathbb{E}\left(\int_{0}^{\tau^{R} \wedge T}\left(|\nabla u|_{L^{2}}^{2}+|u|_{L^{2}}^{\tilde{\sigma}}\right) d s\right) \\
& \leq H\left(u_{0}\right)+C \varepsilon+C \varepsilon\left(\int_{0}^{T} \mathbb{E}\left(\mathbb{1}_{\left[0, \tau^{R} \wedge T\right]}|u(s)|_{\Sigma}^{4}\right) d s\right)+C \beta(\lambda) \gamma T\left|u_{0}\right|_{L^{2}}^{\tilde{\sigma}} .
\end{aligned}
$$

This is not yet sufficient to conclude (3.1) ; we need to compute $\mathbb{E}\left(H(u(\tau))^{2}\right)$ for a stopping time $\tau<\tau^{R}$. We first assume that $\lambda=+1$ and $\sigma<2 / d$. Since, from (3.3),

$$
\begin{aligned}
H(u(\tau))^{2} \leq C H\left(u_{0}\right)^{2}+C\left|2 \varepsilon \int_{0}^{t} \mathbb{1}_{[0, \tau]}\left(\int_{\mathbb{R}^{d}} \nabla u(s) \cdot x \bar{u}(s) d x\right) d W(s)\right|^{2} \\
+C\left(\varepsilon^{2}, \gamma\right)\left(\int_{0}^{\tau}|u|_{\Sigma}^{2} d s\right)^{2}+C \gamma^{2}\left(\int_{0}^{\tau}|u|_{L^{2 \sigma+2}}^{2 \sigma+2} d s\right)^{2} \text { a.s. }
\end{aligned}
$$

we get by taking the expectation and using (3.2) and Lemma 3.1,

$$
\begin{aligned}
& \mathbb{E}\left(H(u(\tau))^{2}\right) \\
& \leq C H\left(u_{0}\right)^{2}+\varepsilon^{2} C \mathbb{E} \int_{0}^{T}\left(\left|\mathbb{1}_{[0, \tau]} \int_{\mathbb{R}^{d}} \nabla u(s) \cdot x \bar{u}(s) d x\right|^{2}\right) d s \\
& \quad+C\left(\varepsilon^{2}, \gamma\right) \mathbb{E}\left(\left.\left.\left|\int_{0}^{\tau}\right| u\right|_{\Sigma} ^{2} d s\right|^{2}\right)+C \gamma^{2} \mathbb{E}\left(\left(\int_{0}^{\tau}|u|_{L^{2 \sigma+2}}^{2 \sigma+2} d s\right)^{2}\right) \\
& \leq C \varepsilon^{2} H\left(u_{0}\right)^{2}+C(\varepsilon, \gamma, T) \int_{0}^{T} \mathbb{E}\left(\mathbb{1}_{[0, \tau]}|u(s)|_{\Sigma}^{4}\right) d s+C\left(\gamma, T,\left|u_{0}\right|_{L^{2}}\right) \\
& \leq C H\left(u_{0}\right)^{2}+C\left(\varepsilon, \gamma, T,\left|u_{0}\right|_{L^{2}}\right) \\
& \quad+C(\varepsilon, \gamma, T) \int_{0}^{T} \mathbb{E}\left(\mathbb{1}_{[0, \tau]} H(u(s))^{2}\right) d s .
\end{aligned}
$$

Hence, we get, for some constant $C=C\left(\varepsilon, \gamma, T,\left|u_{0}\right|_{L^{2}}\right)$ independent of $R$,

$$
\mathbb{E}\left(H(u(t \wedge \tau))^{2}\right) \leq C\left(H\left(u_{0}\right)^{2}+1\right) e^{C T}, \quad \forall t \in[0, T]
$$

Now we go back to (3.7), and combining with (3.2) and (3.8)

$$
\mathbb{E}\left(\sup _{t \in\left[0, \tau^{R} \wedge T\right]} H(u(t))\right) \leq C\left(\varepsilon, \gamma, T,\left|u_{0}\right|_{\Sigma}\right),
$$


which completes the proof in the case $\lambda=1$. Now, if $\lambda=-1$, it suffices to remark that $H(u) \geq 0$ in this case and using that by (3.3) and the Itô Formula,

$$
d\left(H^{2}(u)\right)=2 H(u) d H(u)+4 \varepsilon^{2}\left(\operatorname{Im} \int_{\mathbb{R}^{d}} \nabla u . x \bar{u} d x\right)^{2} d t
$$

we easily get

$$
\begin{aligned}
H^{2}(u(\tau)) \leq & H^{2}\left(u_{0}\right)-4 \varepsilon \int_{0}^{\tau} H(u(s)) \operatorname{Im}\left(\int_{\mathbb{R}^{d}} \nabla u \cdot x \bar{u} d x\right) d W(s) \\
& +4 \varepsilon^{2} \int_{0}^{\tau} H(u(s))|x u(s)|_{L^{2}}^{2} d s-2 \gamma \int_{0}^{\tau} H(u(s))|u(s)|_{\Sigma}^{2} d s \\
& +2 \varepsilon^{2} \int_{0}^{\tau}|u(s)|_{\Sigma}^{4} d s
\end{aligned}
$$

and we conclude as above.

Remark 3.1. In the critical case $\sigma=2 / d$, from the Gagliardo-Nirenberg inequality, it follows that if $u \in \Sigma$ and $|u|_{L^{2}}^{4 / d}<1 /\left(2 C_{d}\right)$ then the estimate $|u|_{\Sigma}^{2} \leq 2 C\left(\left|u_{0}\right|_{L^{2}}\right) H(u)$ holds. Combining this with Lemma 3.1, we conclude Theorem 3 (ii) as above.

\section{Stochastic Virial identity And Blow-UP}

In this section, we derive an identity on the evolution of the "variance" of the solutions of the nonlinear Schrödinger equation (1.3), which is a generalization of the formula in the deterministic case (see [9] for example). Using this variance idendity, we prove Theorem 4. We mainly treat the case $\gamma=0$ with $d=1$ or $d=2$. At the end of the section, we give a remark on the case $\gamma>0$ and $d=1$.

The variance is defined by

$$
I(v)=\int_{\mathbb{R}^{d}}|x|^{2}|v(x)|^{2} d x, \quad \text { for } \quad v \in \Sigma .
$$

Its evolution involves the momentum

$$
G(v)=\operatorname{Im} \int_{\mathbb{R}^{d}} x \bar{v} \nabla v d x, \quad \text { for } \quad v \in \Sigma .
$$

The following proposition describes the evolution of $I$ and $G$.

Proposition 1. Let $\gamma=0$ and $\lambda= \pm 1$. Assume that $u_{0} \in \Sigma$ and $\sigma>0$ if $d=1$ or $u_{0} \in \Sigma^{2}$ and $1 / 2 \leq \sigma \leq 1$ if $d=2$. Then for any stopping time $\tau$ such that $\tau<\tau^{*}\left(u_{0}\right)$ a.s., we have

$$
\begin{aligned}
G(u(\tau)) & =G\left(u_{0}\right)+4 \int_{0}^{\tau} H(u(s)) d s-4 \int_{0}^{\tau}|x u(s)|_{L^{2}}^{2} d s \\
& +\frac{\lambda(2-\sigma d)}{\sigma+1} \int_{0}^{\tau}|u(s)|_{L^{2 \sigma+2}}^{2 \sigma+2} d s-2 \int_{0}^{\tau}|x u(s)|_{L^{2}}^{2} d W(s), \text { a.s. },
\end{aligned}
$$


and

$$
I(u(\tau))=I\left(u_{0}\right)+4 \int_{0}^{\tau} G(u(s)) d s, a . s .
$$

where $H(u)$ is the energy defined by (1.4).

Proposition 1 is obtained thanks to an application of the Itô Formula, using equation (1.3). The computations could be rigorously justified by using the arguments in Section 6.1 of [13]. Again, the assumption $u_{0} \in \Sigma^{2}$ is necessary for this justification, since we need the convergence of the sequence of regurarized solutions to $u$ in $C([0, \tau) ; \Sigma)$.

The following corollary is obtained by combining the above proposition with (3.3).

Corollary 4.1. Under the same assumption as in Proposition 1, we have a.s.

$$
\begin{aligned}
I(u(\tau))= & I\left(u_{0}\right)+4 G\left(u_{0}\right) \tau+8 H\left(u_{0}\right) \tau^{2} \\
& -32 \varepsilon \operatorname{Im} \int_{0}^{\tau} \int_{0}^{s} \int_{0}^{s_{1}} \int_{\mathbb{R}^{d}} \nabla u\left(s_{2}\right) \cdot x \bar{u}\left(s_{2}\right) d x d W\left(s_{2}\right) d s_{1} d s \\
& +32 \varepsilon^{2} \int_{0}^{\tau} \int_{0}^{s} \int_{0}^{s_{1}} I\left(u\left(s_{2}\right)\right) d s_{2} d s_{1} d s-8 \varepsilon \int_{0}^{\tau} \int_{0}^{s} I\left(u\left(s_{1}\right)\right) d W\left(s_{1}\right) d s \\
& -16 \int_{0}^{\tau} \int_{0}^{s} I\left(u\left(s_{1}\right)\right) d s_{1} d s+\frac{4 \lambda(2-\sigma d)}{\sigma+1} \int_{0}^{\tau} \int_{0}^{s}\left|u\left(s_{1}\right)\right|_{L^{2 \sigma+2}}^{2 \sigma+2} d s_{1} d s .
\end{aligned}
$$

We shall now show that, in the critical and supercritical cases, certain initial data with negative energy yield solutions which form a singularity in finite time, with positive probability. In the deterministic case, such kind of statement can be found in $[7,8]$, where the author used a suitable transformation linked to Mehler's formula.

Proof of Theorem 4. Assume that the conclusion of Theorem 4 does not hold; then, $\bar{t}<\tau^{*}\left(u_{0}\right)$ a.s. and we can take $\tau=\bar{t}$ as a stopping time in Corollary 4.1. In that case, (4.3) can be simplified as follows:

$$
\begin{aligned}
I(u(\bar{t}))= & I\left(u_{0}\right)+4 G\left(u_{0}\right) \bar{t}+8 H\left(u_{0}\right) \bar{t}^{2} \\
& -16 \varepsilon \operatorname{Im} \int_{0}^{\bar{t}}(\bar{t}-s)^{2} \int_{\mathbb{R}^{d}} \nabla u(s) \cdot x \bar{u}(s) d x d W(s) \\
& -8 \varepsilon \int_{0}^{\bar{t}}(\bar{t}-s) I(u(s)) d W(s)+\frac{4 \lambda(2-\sigma d)}{\sigma+1} \int_{0}^{\bar{t}}(\bar{t}-s)|u(s)|_{L^{2 \sigma+2}}^{2 \sigma+2} d s \\
& +16 \varepsilon^{2} \int_{0}^{\bar{t}}(\bar{t}-s)^{2} I(u(s)) d s-16 \int_{0}^{\bar{t}}(\bar{t}-s) I(u(s)) d s, \text { a.s. }
\end{aligned}
$$

Note that by the assumption $\varepsilon^{2} \bar{t} \leq 1$, the sum of the two last terms in the right hand side above is nonpositive. Since $\sigma \geq 2 / d$, we are led to

$$
I(u(\bar{t})) \leq I\left(u_{0}\right)+4 G\left(u_{0}\right) \bar{t}+8 H\left(u_{0}\right) \bar{t}^{2}+\varepsilon \int_{0}^{\bar{t}} g(\bar{t}, s) d W(s), a . s .
$$


where

$$
g(\bar{t}, s)=-16(\bar{t}-s)^{2} \operatorname{Im} \int_{\mathbb{R}^{d}} \nabla u(s) \cdot x \bar{u}(s) d x-8(\bar{t}-s) I(u(s)) .
$$

We remark that $\int_{0}^{\bar{t}} g^{2}(\bar{t}, s) d s<+\infty$, a.s. Now, since $I(u(\bar{t})) \geq 0$ a.s., we have

$$
\int_{0}^{\bar{t}} g(\bar{t}, s) d W(s)>\delta>0, \quad \text { a.s. }
$$

and on the other hand,

$$
M(u)=\left(\int_{0}^{u} g(\bar{t}, s) d W(s)\right)_{0 \leq u \leq \bar{t}}
$$

is a local martingale starting at 0 , which is clearly in contradiction with the above inequality.

It is clear that there always exists $u_{0} \in \Sigma$ and $\bar{t}$ with $\varepsilon^{2} \bar{t} \leq 1$ satisfying the assumptions of Theorem 4: it suffices to choose $v \in \Sigma$ with $H(v)<0$ (always possible if $\sigma<2 / d$ ) and to consider $u_{0}=a v$, with $a>0$ sufficiently large.

Remark 4.1. As mentioned in the introduction, it is proved in [13] that for any sufficiently regular $u_{0}$ with $u_{0} \neq 0$, blow-up occurs with positive probability for the corresponding solution, in the attractive supercritical case. Such a result would hold here if we were able to solve the following controlability problem : given $u_{0} \in \Sigma^{2}$, and $T>0$, find $h \in C([0, T] ; \mathbb{R})$ such that the solution of

$$
\left\{\begin{array}{l}
i \partial_{t} v+\Delta v-|x|^{2} v+|v|^{2 \sigma} v=|x|^{2} h(t) v \\
v(0)=u_{0}
\end{array}\right.
$$

satisfies

$$
I(v(T))+G(v(T)) \bar{t}+8 H(v(T)) \vec{t}^{2}<0,
$$

for some $\bar{t}$ with $\varepsilon^{2} \bar{t} \leq 1$. However, we do not know whether this controlability problem has a solution.

Remark 4.2. We may easily adapt as above the arguments of M. Tsutsumi [20] for the case $\gamma>0$. More precisely, assume $d=1, \sigma>2, \lambda=1$, and for some $\bar{t}>0$ and some $b$ with

the initial data $u_{0}$ satisfies

$$
b \leq \min \left\{2\left(\gamma-\varepsilon^{2}\right), \frac{2 \gamma(\sigma+2)}{\sigma-2}\right\},
$$

$$
I\left(u_{0}\right)+\frac{4}{2 \gamma-b}\left(1-e^{-(2 \gamma-b) \bar{t}}\right) G\left(u_{0}\right)<0 ; \quad \text { and } \quad H\left(u_{0}\right) \leq 0,
$$

then collapse occurs with positive probability before the time $\bar{t}$. Note that in the condition above, $b$ need not be positive, so that for any value of $\gamma$ and $\varepsilon>0$, it is possible to find an initial data satisfying the above conditions: indeed, it suffices to consider $u_{0}(x)=$ $\kappa \exp \left(-a x^{2}\right)$ choosing first $a$, and then $\kappa$, sufficiently large. 


\section{Appendix}

In this section we will give a proof of Lemmas 2.1, 2.2 and 2.7. We recall that $u_{n}$ satisfies the equation (2.1) and $W$ is real valued.

Proof of Lemma 2.1. We first prove that $u_{n}$ satisfies

$$
\mathbb{E}\left(\sup _{t \in\left[0, \tau_{n}^{R} \wedge T\right]} H_{n}\left(u_{n}(t)\right)\right) \leq C\left(T,\left|u_{0}\right|_{\Sigma}, R\right),
$$

where

$$
H_{n}(v)=\frac{1}{2}|\nabla v|_{L^{2}}^{2}+\frac{1}{2}|x v|_{L^{2}}^{2}-\int_{\mathbb{R}^{d}} G_{n}(v(x)) d x, \quad G_{n}^{\prime}=g_{n} .
$$

For any $t \in[0, T]$, an application of the Itô formula to the regularized energy (the computations may be justified as in [12]) gives after a few computations

$$
\begin{aligned}
H_{n}\left(u_{n}\right)= & H_{n}\left(u_{0}\right)+\int_{0}^{t}\left\langle H_{n}^{\prime}\left(u_{n}\right),-i \varepsilon x^{2} \theta_{n} u_{n}(s)\right\rangle d W(s) \\
+ & 2 \varepsilon^{2} \int_{0}^{t}\left\{\int_{\mathbb{R}^{d}}\left(\frac{1}{4}\left|\nabla \theta_{n}\right|^{2}|x|^{2}+\theta_{n} x \cdot \nabla \theta_{n}+\theta_{n}^{2}\right)|x|^{2}\left|u_{n}(s)\right|^{2} d x\right\} d s \\
& -\gamma \int_{0}^{t}\left(\left|\nabla u_{n}(s)\right|_{L^{2}}^{2}+\left|x u_{n}(s)\right|_{L^{2}}^{2}+\operatorname{Re} \int_{\mathbb{R}^{d}} g_{n}\left(u_{n}(s)\right) \bar{u}_{n}(s) d x\right) d s \\
\leq & H_{n}\left(u_{0}\right)-\varepsilon \int_{0}^{t}\left\langle H_{n}^{\prime}\left(u_{n}\right), i x^{2} \theta_{n} u_{n}(s)\right\rangle d W(s) \\
& +C \varepsilon^{2} \int_{0}^{t}\left|x u_{n}(s)\right|_{L^{2}}^{2} d s+|\lambda| \gamma \int_{0}^{t}\left|u_{n}(s)\right|_{L^{2 \sigma+2}}^{2 \sigma+2} d s .
\end{aligned}
$$

Here we remark that if $t \leq T \wedge \tau_{n}^{R}$,

$$
\frac{1}{2}\left|u_{n}(t)\right|_{\Sigma}^{2}-\frac{|\lambda|}{2 \sigma+2} R^{2 \sigma+2} \leq H_{n}\left(u_{n}(t)\right) \leq \frac{1}{2}\left|u_{n}(t)\right|_{\Sigma}^{2}+\frac{|\lambda|}{2 \sigma+2} R^{2 \sigma+2} .
$$

Taking the expectation of the supremum of $H_{n}\left(u_{n}\right)$ on $\left[0, T \wedge \tau_{n}^{R}\right]$, and using the martingale inequality of Theorem 3.14 in [10]), we easily get

$$
\begin{aligned}
& \mathbb{E}\left(\sup _{t \in\left[0, T \wedge \tau_{n}^{R}\right]} H_{n}\left(u_{n}(t)\right)\right) \leq H_{n}\left(u_{0}\right)+C T^{1 / 2}\left(1+T^{1 / 2}\right) \mathbb{E}\left(\sup _{t \in\left[0, T \wedge \tau_{n}^{R}\right]}\left|u_{n}(t)\right|_{\Sigma}^{2}\right) \\
&+|\lambda| \gamma T R^{2 \sigma+2} \\
& \leq H_{n}\left(u_{0}\right)+C T^{1 / 2}\left(1+T^{1 / 2}\right) \mathbb{E}\left(\sup _{t \in\left[0, T \wedge \tau_{n}^{R}\right]} H_{n}\left(u_{n}(t)\right)\right) \\
&+C T^{1 / 2}\left(1+T^{1 / 2}\right) \frac{|\lambda|}{\sigma+1} R^{2 \sigma+2}+|\lambda| \gamma T R^{2 \sigma+2} . \\
& 21
\end{aligned}
$$


Taking $T$ small enough, and then reiterating the argument on $\left[T \wedge \tau_{n}^{R}, 2 T \wedge \tau_{n}^{R}\right]$, $\left[2 T \wedge \tau_{n}^{R}, 3 T \wedge \tau_{n}^{R}\right]$, etc., we obtain (5.1) for any $T>0$, which immediately leads to

$$
\mathbb{E}\left(\sup _{t \in\left[0, \tau_{n}^{R} \wedge T\right]}\left|u_{n}(t)\right|_{\Sigma}^{2}\right) \leq C\left(T,\left|u_{0}\right|_{\Sigma}, R\right)
$$

Proof of Lemma 2.2. It follows easily from the Itô fomula that

$$
\left|u_{n}(t)\right|_{L^{2}}^{2}=\left|u_{0}\right|_{L^{2}}^{2}-2 \gamma\left|u_{n}(t)\right|_{L^{2}}^{2} \quad \text { a.s. }
$$

for any $t \in[0, T]$, hence

$$
\mathbb{E}\left(\left|u_{n}(t)\right|_{C\left([0, T] ; L^{2}\right)}^{2 l}\right) \leq\left|u_{0}\right|_{L^{2}}^{2 l}, \quad l \in \mathbb{N}
$$

We now use the integral form of the equation (2.1) satisfied by $u_{n}$ to get an estimate on its modulus of continuity. Let $\tau_{1}, \tau_{2} \geq 0$ with $\tau_{n}^{R} \geq \tau_{2}>\tau_{1}$. Integrating from $\tau_{1}$ to $\tau_{2}$,

$$
\begin{aligned}
u_{n}\left(\tau_{2}\right)-u_{n}\left(\tau_{1}\right)= & i \int_{\tau_{1}}^{\tau_{2}}\left(\Delta u_{n}(s)-|x|^{2} u_{n}(s)\right) d s-\frac{\varepsilon^{2}}{2} \int_{\tau_{1}}^{\tau_{2}} \theta_{n}^{2}|x|^{4} u_{n}(s) d s \\
& +i \lambda \int_{\tau_{1}}^{\tau_{2}}\left|u_{n}(s)\right|^{2 \sigma} u_{n}(s) d s-i \varepsilon \int_{\tau_{1}}^{\tau_{2}}|x|^{2} \theta_{n} u_{n}(s) d W(s)
\end{aligned}
$$

The terms of the right hand side of (5.3) except the last term are in $C^{1}\left(\left[0, T \wedge \tau_{n}^{R}\right] ; \Sigma^{-4}\right)$. In fact, by (5.2),

$$
\mathbb{E}\left(\sup _{t \in\left[0, T \wedge \tau_{n}^{R}\right]}\left|\left(\Delta-|x|^{2}-\frac{\varepsilon^{2}}{2} \theta_{n}^{2}|x|^{4}\right) u_{n}\right|_{\Sigma^{-4}}^{2}\right) \leq C \mathbb{E}\left(\left|u_{n}\right|_{C\left(\left[0, T \wedge \tau_{n}^{R}\right] ; L^{2}\right)}^{2}\right) \leq C,
$$

where $C$ does not depend on $n$.

Concerning the third term, we obtain, using the embedding $\Sigma^{-4} \subset H^{-1} \subset L^{(2 \sigma+2) /(2 \sigma+1)}$,

$$
\mathbb{E}\left(\left.\left.\sup _{0 \leq \tau_{1} \leq \tau_{2} \leq \tau_{n}^{R}}\left|\int_{\tau_{1}}^{\tau_{2}}\right| u_{n}(s)\right|^{2 \sigma} u_{n}(s) d s\right|_{\Sigma^{-4}} ^{2}\left|\tau_{1}-\tau_{2}\right|^{-2}\right) \leq C(R) \mathbb{E}\left(\sup _{s \in\left[0, \tau_{n}^{R}\right]}\left|u_{n}(s)\right|_{\Sigma}^{2}\right)
$$

which is bounded independently of $n$, by Lemma 2.1 .

Therefore, it suffices to verify that the forth term of the right hand side of (5.3) is of $\alpha$-Hölder class, i.e., for $\alpha>0$,

$$
\mathbb{E}\left(\left.\left.\sup _{0 \leq \tau_{1} \leq \tau_{2} \leq \tau_{n}^{R}}\left|\int_{\tau_{1}}^{\tau_{2}} i \varepsilon\right| x\right|^{2} \theta_{n} u_{n}(s) d W(s)\right|_{\Sigma^{-4}} ^{2}\left|\tau_{1}-\tau_{2}\right|^{-2 \alpha}\right) \leq C
$$


where $C$ does not depend on $n$. It follows from the Bürkholder-Davis-Gundy inequality,

$$
\begin{aligned}
\mathbb{E}\left(\left.\left.\left|\int_{\tau_{1}}^{\tau_{2}}\right| x\right|^{2} \theta_{n} u_{n}(s) d W(s)\right|_{\Sigma^{-4}} ^{4}\right) & \leq C \mathbb{E}\left(\left(\left.\left.\int_{\tau_{1}}^{\tau_{2}}|| x\right|^{2} \theta_{n} u_{n}(s)\right|_{\Sigma^{-4}} ^{2} d s\right)^{2}\right) \\
& \leq C \mathbb{E}\left(\left.\left.\sup _{s \in\left[0, \tau_{n}^{R}\right]}|| x\right|^{2} \theta_{n} u_{n}(s)\right|_{\Sigma^{-4}} ^{4}\right)\left|\tau_{2}-\tau_{1}\right|^{2} \\
& \leq C\left|u_{0}\right|_{L^{2}}^{4}\left|\tau_{2}-\tau_{1}\right|^{2} .
\end{aligned}
$$

We have used (5.2) in the last inequality. Applying then the Kolmogorov criterion, we get that the forth term in (5.3) is a.s. in $C^{\alpha}\left(\left[0, T \wedge \tau_{n}^{R}\right] ; \Sigma^{-4}\right)$ for $\alpha<1 / 4$.

Proof of Lemma 2.7. We recall that $d=2$. By Lemma 2 in [6], for any $N \in \mathbb{N}$, there exists a constant $C(N)>0$ such that for any $v \in \Sigma^{2}$ with $|v|_{\Sigma} \leq N$,

$$
|v|_{L^{\infty}} \leq C(N)\left(1+\sqrt{\log \left(1+|v|_{\Sigma^{2}}^{2}\right)}\right) .
$$

Let $F(v)=|v|_{\Sigma^{2}}^{2}$. We apply the Itô formula to $1+\log \left(1+\left|u_{n}\right|_{\Sigma^{2}}^{2}\right)$. Then, for any stopping time $\tau$ with $\tau \leq \hat{\tau}_{n}^{N} \wedge T$ a.s., we have

$$
\begin{aligned}
& 1+\log \left(1+\left|u_{n}(\tau)\right|_{\Sigma^{2}}^{2}\right) \\
\leq & 1+\log \left(1+\left|u_{0}\right|_{\Sigma^{2}}^{2}\right)-\varepsilon \int_{0}^{\tau} \frac{1}{1+\left|u_{n}(s)\right|_{\Sigma^{2}}^{2}}\left\langle F^{\prime}\left(u_{n}\right), i \theta_{n}|x|^{2} u_{n}(s)\right\rangle d W(s) \\
& +C(\varepsilon) T+C \int_{0}^{\tau} \frac{1}{1+\left|u_{n}(s)\right|_{\Sigma^{2}}^{2}}\left|u_{n}(s)\right|_{L^{\infty}}^{2 \sigma}\left|u_{n}(s)\right|_{\Sigma^{2}}^{2} d s \\
\leq & 1+\log \left(1+\left|u_{0}\right|_{\Sigma^{2}}^{2}\right)-\varepsilon \int_{0}^{\tau} \frac{1}{1+\left|u_{n}(s)\right|_{\Sigma^{2}}^{2}}\left\langle F^{\prime}\left(u_{n}\right), i \theta_{n}|x|^{2} u_{n}(s)\right\rangle d W(s) \\
& +C(\varepsilon) T+C(N) \int_{0}^{\tau}\left(1+\log \left(1+\left|u_{n}(s)\right|_{\Sigma^{2}}^{2}\right)\right) d s .
\end{aligned}
$$

In the first inequality we have estimated the nonlinear part in such a way as

$$
\left|\Delta g_{n}\left(u_{n}\right)\right|_{L^{2}} \leq C\left|u_{n}\right|_{L^{\infty}}^{2 \sigma}\left|u_{n}\right|_{\Sigma^{2}}^{2}, \quad \sigma \geq 1 / 2
$$

where the constant $C$ is independent of $n$. The last inequality follows from $\sigma \leq 1$ and (5.4). 
Taking the expectation of the supremum of $1+\log \left(1+\left|u_{n}(t)\right|_{\Sigma^{2}}^{2}\right)$ on $\left[0, \hat{\tau}_{n}^{N} \wedge T\right]$, and using a martingale inequality as in the proof of Lemma 2.1,

$$
\begin{gathered}
\mathbb{E}\left(\sup _{t \in\left[0, \hat{\tau}_{n}^{N} \wedge T\right]}\left(1+\log \left(1+\left|u_{n}(t)\right|_{\Sigma^{2}}^{2}\right)\right)\right) \\
\leq 1+\log \left(1+\left|u_{0}\right|_{\Sigma^{2}}^{2}\right)+\varepsilon \mathbb{E}\left(\sup _{t \in\left[0, \hat{\tau}_{n}^{N} \wedge T\right]}\left|\int_{0}^{t} \frac{1}{1+\left|u_{n}(s)\right|_{\Sigma^{2}}^{2}}\left\langle F^{\prime}\left(u_{n}\right), i \theta_{n}|x|^{2} u_{n}(s)\right\rangle d W(s)\right|\right) \\
+C(\varepsilon) T+C(N) T \mathbb{E}\left(\sup _{t \in\left[0, \hat{\tau}_{n}^{N} \wedge T\right]}\left(1+\log \left(1+\left|u_{n}(t)\right|_{\Sigma^{2}}^{2}\right)\right)\right) \\
\leq C\left(\left|u_{0}\right|_{\Sigma^{2}}\right)+\varepsilon C \mathbb{E}\left\{\left(\int_{0}^{\hat{\tau}_{n}^{N} \wedge T}\left(\frac{\left|u_{n}(s)\right|_{\Sigma^{2}}^{2}}{1+\left|u_{n}(s)\right|_{\Sigma^{2}}^{2}}\right)^{2} d s\right)^{1 / 2}\right\} \\
+C(\varepsilon) T+C(N) T \mathbb{E}\left(\sup _{t \in\left[0, \hat{\tau}_{n}^{N} \wedge T\right]}\left(1+\log \left(1+\left|u_{n}(t)\right|_{\Sigma^{2}}^{2}\right)\right)\right) \\
\leq C\left(\left|u_{0}\right|_{\Sigma^{2}}\right)+C(\varepsilon) T^{1 / 2}\left(1+T^{1 / 2}\right)+C(N) T \mathbb{E}\left(\sup _{t \in\left[0, \hat{\tau}_{n}^{N} \wedge T\right]}\left(1+\log \left(1+\left|u_{n}(t)\right|_{\Sigma^{2}}^{2}\right)\right)\right) .
\end{gathered}
$$

Hence, we have, for sufficiently small $T>0$,

$$
\mathbb{E}\left(\sup _{t \in\left[0, \hat{\tau}_{n}^{N} \wedge T\right]}\left(1+\log \left(1+\left|u_{n}(t)\right|_{\Sigma^{2}}^{2}\right)\right)\right) \leq C\left(T,\left|u_{0}\right|_{\Sigma^{2}}, N\right) .
$$

Reiterating the argument on $\left[\hat{\tau}_{n}^{N} \wedge T, \hat{\tau}_{n}^{N} \wedge 2 T\right],\left[\hat{\tau}_{n}^{N} \wedge 2 T, \hat{\tau}_{n}^{N} \wedge 3 T\right]$, etc., we complete the proof of (2.10).

\section{REFERENCES}

1. F.Kh. Abdullaev, B.B Baizakov and V.V. Konotop, "Dynamics of a Bose-Einstein condensate in optical trap," in: Nonlinearity and Disorder : Theory and Applications, edited by F.Kh. Abdullaev, O. Bang and M.P. Soerensen, NATO Science Series vol. 45, Kluwer Dodrecht, 2001.

2. F.Kh. Abdullaev, J.C. Bronski and R.M Galimzyanov, "Dynamics of a trapped 2D Bose-Einstein condensate with periodically and randomly varying atomic scattering length," Physica D 184 (2003) 319-332.

3. F.Kh. Abdullaev, J.C. Bronski and G. Papanicolaou, "Soliton perturbations and the random Kepler problem," Physica D 135 (2000) 369-386.

4. S. Albeverio, V.N.Kolkol'tsov and O.G. Smolyanov, "Continuous Quantum mesurement : local and global approaches," Rev. Math. Phys. 9, (1997), 907-920.

5. O. Bang, P.L. Christiansen, F. If, K.O. Rasmussen, "Temperature effects in a nonlinear model of monolayer Scheibe aggregates," Phys. Rev. E 49, (1994), 4627-4636.

6. H. Brezis and T. Gallouet, "Nonlinear Schrödinger evolution equations," Nonlinear Anal., Theory, Methods \& Applications. 4, (1980), 677-681. 
7. R. Carles, "Remarks on nonlinear Schrödinger equations with harmonic potential," Ann. Henri Poincaré. 3 (2002) 757-772.

8. R. Carles, "Nonlinear Schrödinger equations with repulsive harmonic potential and applications," SIAM. J. Math. Anal. 35 (2003) 823-843.

9. T.Cazenave, "Semilinear Schrödinger equations," Courant Lecture Notes in Mathematics, 10, American Mathematical Society, Courant Institute of Mathematical Sciences, 2003.

10. G. Da Prato and J. Zabczyk, "Stochastic equations in infinite dimensions," Encyclopedia of Mathematics and its Applications; Cambridge University Press: Cambridge, England, 1992.

11. A. de Bouard and A. Debussche, "The stochastic nonlinear Schrödinger equation with multicative noise," Commun. Math. Phys. 205 (1999) 161-181.

12. A. de Bouard and A. Debussche, "The stochastic nonlinear Schrödinger equation in $H^{1}$," Stochastic analysis and applications 21 (2003) 97-126.

13. A. de Bouard and A. Debussche, "Blow up for the stochastic nonlinear Schrödinger equation with multicative noise," Ann. Probab. 33 (2005) 1078-1110.

14. A. de Bouard and A. Debussche, "A semi-discrete scheme for the stochastic nonlinear Schrödinger equation," Numer.Math. 96 (2004) 733-770.

15. R.P. Feynman and A.R. Hibbs, "Quantum mechanics and path integrals," International Series in Pure and Applied Physics, McGraw-Hill Publishing Company, Maidenhead, Berksh, 1965.

16. G. Fibich and G. Papanicolau, "Self-focusing in the perturbed and unperturbed nonlinear Schrödinger equation in critical dimension," SIAM. J. Appl. Math. 60 (1999) 183-240.

17. J. Garnier, F.Kh. Abdullaev and B.B. Baizakov, "Collapse of a Bose-Einstein condensate induced by fluctuations of the laser intensity," Phys. Rev. A 69 (2004) 053607, 369-386.

18. I. Gyöngy and N.V.Krylov, "Existence of strong solutions for Itô's stochastic equations via approximations," Probab. Theory Relat. Fields 105 (1996) 143-158.

19. Y.-G. Oh, "Cauchy problem and Ehrenfest's law of nonlinear Schrödinger equations with potentials," J. Differential Equations 81 (1989) 255-274.

20. M. Tsutsumi, "Nonexistence of global solutions to the Cauchy problem for the damped nonlinear Schrödinger equations," Academic Press, 1975. SIAM. J. Math. Anal. 15 (1984) 357-366.

21. K.Yajima and G.P. Zhang, "Smoothing property for Schrödinger equations with potential superquadratic at infinity," Commun. Math. Phys. 221 (2001) 573-590.

22. K.Yajima and G.P. Zhang, "Local smoothing property and Strichartz inequality for Schrödinger equations with potentials superquadratic at infinity," J. Diff. Eqs. 202 (2004) 81-110. 